\title{
HELICAL MINIMAL IMMERSIONS OF COMPACT RIEMANNIAN MANIFOLDS INTO A UNIT SPHERE
}

BY

KUNIO SAKAMOTO

\begin{abstract}
ABSTRACr. An isometric immersion of a Riemannian manifold $M$ into a Riemannian manifold $\bar{M}$ is called helical if the image of each geodesic has constant curvatures which are independent of the choice of the particular geodesic. Suppose $M$ is a compact Riemannian manifold which admits a minimal helical immersion of order 4 into the unit sphere. If the Weinstein integer of $M$ equals that of one of the projective spaces, then $M$ is isometric to that projective space with its canonical metric.
\end{abstract}

0. Introduction. In [2], Besse constructed a minimal immersion with a nice property of a strongly harmonic manifold into a sphere. This nice property is that the images of geodesics of the strongly harmonic manifold are of constant curvatures as curves in the sphere, and the curvatures and the osculating orders are independent of geodesics. Immersions with such a property are said to be helical (cf. [8]). Sakamoto [8] studied a helical immersion of a Riemannian manifold $M$ into a unit sphere. The result is that if $M$ is compact, then it is a Blaschke manifold and, moreover, if the helical immersion is minimal, then $M$ is a globally harmonic manifold. It is well known that if a globally harmonic manifold is compact and simply connected, then it is a strongly harmonic manifold (Michel's theorem, cf. [2]). Thus, we can declare that the theory of helical minimal immersions of compact simply connected Riemannian manifolds into a unit sphere is a submanifold version of strongly harmonic manifold theory.

Let $f: M \rightarrow S(1)$ be a helical immersion of a compact Riemannian manifold $M$ into a unit sphere $S(1)$. Since $M$ is a Blaschke manifold, all geodesics of $M$ are simply closed and of the same length. Furthermore, if we denote the cut-locus of $x \in M$ by $\operatorname{Cut}(x)$, then the unit tangent vectors at $x$ of geodesics emanating from $x$ and entering to $y \in \operatorname{Cut}(x)$ compose a great sphere in the unit tangent sphere at $x$. The dimension of the great sphere is independent of $x$ and it is equal to $0,1,3,7$, or $n-1(n=\operatorname{dim} M)$, which is the index of the first conjugate point $y$ of $x$ (cf. [2], Proposition 5.39 and Theorem 7.23). Geodesics are also helical in the Euclidean

Received by the editors March 15, 1984 and, in revised form, June 20, 1984.

1980 Mathematics Subject Classification. Primary 53C40; Secondary 53C42.

Key words and phrases. Helical immersions, strongly harmonic manifolds, geodesics, Blaschke structure, cut loci, second fundamental forms. 
space $E$ in which $S(1)$ is naturally embedded. Thus, it is easy to find the equation of geodesics in $E$ if we are given a certain initial condition which consists of the second fundamental form of $f$ and its covariant derivatives. So we can express the immersion $f$ in terms of geodesic polar coordinates by using the second fundamental form and its covariant derivatives at the center (cf. Theorem 1.2). Moreover, we can compute all normal Jacobi fields (precisely, their images by $f$ ). Therefore, we are interested in the study of the property of the second fundamental form derived from Blaschke structure.

Helical immersions of order 2 into a sphere are planar geodesic immersions which are completely classified in [5 and 7]. Helical minimal immersions of order 3 into a sphere were determined by Nakagawa [6] under a more general situation. In the present paper we shall study helical minimal immersions of order 4 into a unit sphere.

The organization of this paper is as follows. We deal with a helical immersion $f$ : $M \rightarrow S(1)$, where $M$ is a compact Riemannian manifold. In $\S 1$ we explain the results obtained in [8] together with notation. In $\$ 2$ we compute all normal Jacobi fields by making use of the expression of the immersion $f$ stated in Theorem 1.2. As a corollary we show that the second fundamental form satisfies equations containing the arc length parameter of the geodesic. In $\$ 3$, we discuss a property of the second fundamental form which is derived from the Blaschke structure. We also characterize the tangent space of the cut-locus and its orthogonal complement (which is a holomorphic section if $M$ is a complex projective space) as eigenspaces of the second fundamental tensor (shape operator) corresponding to some normal vector. In $\S 4$, making use of results obtained in $\S 3$, we show that compact Riemannian manifolds, admitting helical minimal immersions of order 3 into a unit sphere, are isometric to a sphere of constant curvature. The proof is different from Nakagawa [6]. We also show that if the cut-locus degenerates to one point (and hence $M$ is isometric to a sphere of constant curvature because of [2, Theorem D.1, p. 236]), then the order of the helical minimal embedding $f$ must be odd. These results lead us to conjecture that if the order of a helical minimal embedding of a compact Riemannian manifold into a unit sphere is odd, then the manifold will be isometric to a sphere of constant curvature. As the cut-loci of compact rank-one symmetric spaces are totally geodesic submanifolds, we hope that cut-loci are totally geodesic in $M$. Here we give good information about the total-geodesicity of cut-loci. At the end of this section we consider the fiber bundle whose total space is the unit tangent sphere at $x$ and whose base space is the cut-locus $\operatorname{Cut}(x)$ (cf. [2, p. 134]).

If the projection of the fiber bundle is a Riemannian submersion, then we can apply Escobales' result [3] to this submersion. We shall give a certain condition for this application. In $\$ 5$ we consider a helical minimal immersion of order 4 . We prove that if $a=\langle\dot{\gamma}(0), \dot{\gamma}(L)\rangle$ for a unit speed geodesic $\gamma$, where $L$ denotes the distance from $\gamma(0)$ to the cut point and $\langle$,$\rangle , the inner product in E$, is positive, then $M$ is isometric to one of the compact rank-one symmetric spaces. In $\S 6$ we continue to study helical minimal immersions of order 4 and compute the Weinstein integer of $M$. We show that if the Weinstein integer of $M$ is equal to that of a certain compact 
rank-one symmetric space and if $M$ admits a helical minimal imbedding of order 4 into the unit sphere, then $M$ is isometric to that rank-one symmetric space.

The author wishes to express his hearty thanks to Professor S. Ishihara for his constant encouragement and valuable suggestions.

1. Preliminaries. In this paper, the differentiability of all geometric objects will be $C^{\infty}$. Let $M$ be a connected complete Riemannian manifold and $f: M \rightarrow \bar{M}$ an isometric immersion into a Riemannian manifold $\bar{M}$. Let $\sigma$ be a curve $I \rightarrow \bar{M}$ parametrized by arc length $s$. Let $\sigma^{(1)}=\dot{\sigma}$ be the unit tangent vector and put $\kappa_{1}=\left\|\nabla_{\dot{\sigma}} \sigma^{(1)}\right\|$. If $\kappa_{1}$ vanishes on $I$, then $\sigma$ is said to be of order 1 . If $\kappa_{1}$ is not identically zero, then we define $\sigma^{(2)}$ by $\nabla_{\dot{\sigma}} \sigma^{(1)}=\kappa_{1} \sigma^{(2)}$ on the set $I_{1}=\{s \in I$ : $\left.\kappa_{1}(s) \neq 0\right\}$. Put $\kappa_{2}=\left\|\nabla_{\dot{\sigma}} \sigma^{(2)}+\kappa_{1} \sigma^{(1)}\right\|$. If $\kappa_{2} \equiv 0$ on $I_{1}$, then $\sigma$ is said to be of order 2. If $\kappa_{2}$ is not identically zero on $I_{1}$, then we define $\sigma^{(3)}$ by $\nabla_{\dot{\sigma}} \sigma^{(2)}=-\kappa_{1} \sigma^{(1)}+\kappa_{2} \sigma^{(3)}$. Inductively we put

$$
\kappa_{d}=\left\|\nabla_{\dot{\sigma}} \sigma^{(d)}+\kappa_{d-1} \sigma^{(d-1)}\right\|,
$$

and if $\kappa_{d} \equiv 0$ on $I_{d-1}$, then $\sigma$ is said to be of order $d$. Let $\gamma$ be an arbitrary geodesic of $M$. If the curve $\sigma=f \circ \gamma$ in $\bar{M}$ is of order $d$ and has constant curvatures which do not depend on $\gamma$, then $f: M \rightarrow \bar{M}$ is called a helical immersion of order $d$. In the sequel, the ambient manifold $\bar{M}$ will be a unit sphere $S(1)$.

Let $\iota: S(1) \rightarrow E$ be the canonical inclusion, where $E$ is a Euclidean space whose origin is the center of $S(1)$. In [8] we showed that $\tilde{f}=\imath \circ f: M \rightarrow E$ is also a helical immersion of order $d^{*}$, where

$$
d^{*}= \begin{cases}d & \text { if } d \text { is an even integer } \\ d+1 & \text { if } d \text { is an odd integer }\end{cases}
$$

The curvatures $\lambda_{1}, \ldots, \lambda_{d^{*}-1}$ of $\tau=\tilde{f} \circ \gamma$ are rational functions of curvatures $\kappa_{1}, \ldots, \kappa_{d-1}$ of $\sigma$ (cf. [8, Corollary 4.2]). We computed in [8] the Frenet vectors of the curve $\tau$. If we denote the second fundamental form of the immersion $f: M \rightarrow S(1)$ by $H$ and the van der Waerden-Bortolotti covariant differentiation with respect to $f$ by $D$, then they are given as follows:

THEOREM 1.1. Let $\gamma: \mathbf{R} \rightarrow M$ be a unit speed geodesic such that $\gamma(0)=x$ and $\dot{\gamma}(0)=X$. Then Frenet vectors $\tau^{(j)}(X), j=1, \ldots, d$, at $x$ of the curve $\tau$ are given by

$$
\begin{aligned}
\tau^{(1)}(X) & =\tilde{f}_{*} X \\
\tau^{(j)}(X) & =\left(\lambda_{1} \cdots \lambda_{j-1}\right)^{-1}\left[-b_{j 2} f(x)+\sum b_{j i}\left(D^{i-2} H\right)\left(X^{i}\right)\right] \quad \text { if } j \text { is even, } \\
\tau^{(j)}(X) & =\left(\lambda_{1} \cdots \lambda_{j-1}\right)^{-1} \sum b_{j i}\left(D^{i-2} H\right)\left(X^{i}\right) \text { if } j \text { is odd, }
\end{aligned}
$$

where the index $i$ in the summation runs over the range $\{2,4, \ldots, j\}$ and $\{3,5, \ldots, j\}$, respectively. When $d$ is an odd integer, Frenet vector $\tau^{(d+1)}(X)$ is given by

$$
\tau^{(d+1)}(X)=\left(\lambda_{1} \cdots \lambda_{d}\right)^{-1}\left[-b_{d+12} \tilde{f}(x)+\sum\left(b_{d+1 k}-a_{d+1 k}\right)\left(D^{k-2} H\right)\left(X^{k}\right)\right],
$$

where the index $k$ in the summation runs over the range $\{2,4, \ldots, d-1\}$. The coefficients $b_{j i}\left(\right.$ resp. $\left.a_{d+1 k}\right)$ are polynomials of $\lambda_{1}, \ldots, \lambda_{d^{*-1}}\left(\right.$ resp. $\left.\kappa_{1}, \ldots, \kappa_{d-1}\right)$. 
Let $\Lambda$ be the Frenet matrix

$$
\left(\begin{array}{ccccc}
0 & -\lambda_{1} & & & \\
\lambda_{1} & 0 & -\lambda_{2} & & \\
& \ddots & \ddots & \ddots & \\
& & \ddots & \ddots & -\lambda_{d^{*}-1} \\
& & & \lambda_{d^{*}-1} & 0
\end{array}\right)
$$

and ${ }^{t}\left(f_{1}(s), \ldots, f_{d^{*}}(s)\right)$ the first column of the matrix $\left(e^{s \Lambda}-I\right) \Lambda^{-1}$. Thus, functions $f_{i}$ defined on $\mathbf{R}$ satisfy

$$
\begin{aligned}
f_{1}^{\prime} & =1-\lambda_{1} f_{2}, \\
f_{i}^{\prime} & =\lambda_{i-1} f_{i-1}-\lambda_{i} f_{i+1} \quad\left(2 \leqslant i \leqslant d^{*}-1\right), \\
f_{d^{*}}^{\prime} & =\lambda_{d^{*}-1} f_{d^{*}-1} .
\end{aligned}
$$

We see easily from the definition that $f_{1}$ is an odd function. Therefore if $i$ is odd (resp. even), then $f_{i}$ is an odd (resp. even) function. For the sake of convenience, we shall adopt the following notation:

$$
\begin{gathered}
\tilde{\tau}^{(j)}(X)=\left(\lambda_{1} \cdots \lambda_{j-1}\right)^{-1} \sum b_{j i}\left(D^{i-2} H\right)\left(X^{i}\right) \quad \text { if } j(\leqslant d) \text { is even, } \\
\tilde{\tau}^{(d+1)}(X)=\left(\lambda_{1} \cdots \lambda_{d}\right)^{-1} \sum\left(b_{d+1 k}-a_{d+1 k}\right)\left(D^{k-2} H\right)\left(X^{k}\right) \text { if } d \text { is odd, }
\end{gathered}
$$

and

$$
\begin{aligned}
& \xi(s ; X)=\sum_{j: \text { even }} f_{j}(s) \tilde{\tau}^{(j)}(X), \\
& \zeta(s ; X)=\sum_{j: \text { odd } \geqslant 3} f_{j}(s) \tau^{(j)}(X)
\end{aligned}
$$

for arbitrary unit vector $X$ tangent to $M$. In [8] we showed

Theorem 1.2. For $s \in \mathbf{R}$ and $X \in U_{x} M$ (unit tangent sphere at $x$ ), we have

$$
\tilde{f}\left(\exp _{x} s X\right)=F(s) \tilde{f}(x)+f_{1}(s) \tilde{f}_{*} X+\xi(s ; X)+\zeta(s ; X),
$$

where

$$
F(s)=1-\sum_{j: \text { even }} c_{j} f_{j}(s), \quad c_{j}=\frac{\lambda_{2} \lambda_{4} \cdots \lambda_{j-2}}{\lambda_{1} \lambda_{3} \cdots \lambda_{j-1}} .
$$

Therefore, we see that position vectors $\tilde{f}(x)$ and $\tilde{f}(y)$ satisfy $\langle\tilde{f}(x), \tilde{f}(y)\rangle=$ $F(\delta(x, y))$ for every $x, y \in M$ where $\langle$,$\rangle is the inner product on E$ and $\delta$ denotes the distance function on $M$. From this fact, we obtain

THEOREM 1.3 [8]. If a compact Riemannian manifold $M$ admits a helical immersion $f: M \rightarrow S(1)$, then $M$ is a Blaschke manifold.

COROLlARY 1.4. If a helical immersion $f: M \rightarrow S(1)$ is not an embedding, then the manifold $M$ is diffeomorphic to a sphere and $f$ is invariant under the action of antipody of the Blaschke structure on $M$ described in the above theorem; in this case the map $f$ is two-sheeted on $f(M)$ which is diffeomorphic to $\mathbf{R} P^{n}$. Moreover, $M$ is simply connected except for the case that $M$ is diffeomorphic to $\mathbf{R} P^{n}$. 
If an isometric immersion $f: M \rightarrow S(1)$ is minimal, then it is well known that height functions are eigenfunctions of the Laplace operator corresponding to the eigenvalue $n=\operatorname{dim} M$. Thus, we obtain

THEOREM 1.5 [8]. If a Riemannian manifold $M$ admits a helical minimal immersion $f: M \rightarrow S(1)$, then $M$ is a globally harmonic manifold. Furthermore, if $M$ is compact and simply connected, then $M$ is a strongly harmonic manifold.

Conversely, if $M$ is a strongly harmonic manifold, then $M$ admits a helical minimal immersion into a unit sphere (cf. [2, Theorem 6.99]). Thus, the theory of helical minimal immersions is a submanifold version of harmonic manifolds.

2. Jacobi fields. In the sequel, $f: M \rightarrow S(1)$ will be a helical embedding. By virtue of Corollary 1.4 we may assume that $f$ is an embedding. Thus we shall identify $x \in M$ with $\tilde{f}(x) \in E$. The tangent space $T_{x} M$ will be identified with the subspace $\tilde{f}_{*}\left(T_{x} M\right)$ of $T_{x} E \approx E$. Before computing Jacobi fields, we introduce some notation to avoid complexity. Let $X$ and $V$ be orthonormal vectors at $x$ tangent to $M$. Define the following normal vectors at $x$ :

$$
\begin{aligned}
& \xi_{X}(s ; V)=\left.\frac{d}{d \theta} \xi(s ; \cos \theta X+\sin \theta V)\right|_{\theta=0}, \\
& \zeta_{X}(s ; V)=\left.\frac{d}{d \theta} \zeta(s ; \cos \theta X+\sin \theta V)\right|_{\theta=0}, \\
& (D \xi)(s ; V ; X)=\sum f_{j}(s)\left(D \tilde{\tau}^{(j)}\right)(V ; X), \\
& (D \zeta)(s ; V ; X)=\sum f_{j}(s)\left(D \tau^{(j)}\right)(V ; X),
\end{aligned}
$$

where $D \tilde{\tau}^{(j)}(V ; X)$ is defined by

$$
D \tilde{\tau}^{(j)}(V ; X)=\left(\lambda_{1} \cdots \lambda_{j-1}\right)^{-1} \sum b_{j i}\left(D^{i-1} H\right)(V, X, \ldots, X)
$$

for $j=2,4, \ldots \leqslant d$; if $d$ is an odd integer, then

$$
D \tilde{\tau}^{(d+1)}(V ; X)=\left(\lambda_{1} \cdots \lambda_{d}\right)^{-1} \sum\left(b_{d+1 k}-a_{d+1 k}\right)\left(D^{k-1} H\right)(V, X, \ldots, X),
$$

and if $j$ is odd, then $D \tau^{(j)}(V ; X)$ is defined in a similar way.

Let us now compute Jacobi fields along a unit speed geodesic $\gamma$. Let $x=\gamma(0)$, $X=\dot{\gamma}(0)$ and let $V$ be a unit tangent vector orthogonal to $X$. In Theorem 1.2 we exchange $X$ for $\cos \theta X+\sin \theta V$. Then we have a variation of geodesics. Furthermore, if $X^{*}(t)$ is a vector field parallel to $X$ along a curve $\beta(t)$ parametrized by arc length such that $\beta(0)=x$ and $\dot{\beta}(0)=V$, then each geodesic which issues from $\beta(t)$ and is tangent to $X^{*}(t)$ forms another variation. These variations yield all normal Jacobi fields along $\gamma$. We have

THeORem 2.1. Let $J_{V}$ and $J_{V}^{*}$ be normal Jacobi fields along $\gamma$ such that $J_{V}(0)=0$, $\nabla_{\dot{\gamma}} J_{V}(0)=V$ and $J_{V}^{*}(0)=V, \nabla_{\dot{\gamma}} J_{V}^{*}(0)=0$, respectively. Then they are given by

$$
\begin{aligned}
& J_{V}(s)=f_{1}(s) V+\xi_{X}(s ; V)+\zeta_{X}(s ; V), \\
J_{V}^{*}(s)= & F(s) V-A_{\xi(s ; X)} V-A_{\zeta(s ; X)} V \\
+ & f_{1}(s) H(V, X)+(D \xi)(s ; V ; X)+(D \zeta)(s ; V ; X),
\end{aligned}
$$


where $A_{\xi(s ; X)}$ (resp. $A_{\xi(s ; X)}$ ) denotes the second fundamental tensor corresponding to the normal vector $\xi(s ; X)($ resp. $\zeta(s ; X))$.

Proof. Consider the variation $(s, \theta) \mapsto \exp _{x} s(\cos \theta X+\sin \theta V)$. Then we have, from Theorem 1.2,

$$
\begin{aligned}
J_{V}(s)= & \frac{d}{d \theta}\left[F(s) x+f_{1}(s)(\cos \theta X+\sin \theta V)\right. \\
& +\xi(s ; \cos \theta X+\sin \theta V)+\zeta(s ; \cos \theta X+\sin \theta V)]\left.\right|_{\theta=0} \\
= & f_{1}(s) V+\xi_{X}(s ; V)+\zeta_{X}(s ; V) .
\end{aligned}
$$

Next let $\beta(t)$ and $X^{*}(t)$ be as above. We then consider the variation $(s, t) \mapsto$ $\exp _{\beta(t)} s X^{*}(t)$. Theorem 1.2 implies that

$$
\begin{aligned}
J_{V}^{*}(s)= & \left.\frac{d}{d t}\left[F(s) \beta(t)+f_{1}(s) X^{*}(t)+\xi\left(s ; X^{*}(t)\right)+\zeta\left(s ; X^{*}(t)\right)\right]\right|_{t=0} \\
= & F(s) V+f_{1}(s) H(X, V)-A_{\xi(x ; X)} V+(D \xi)(s ; V ; X) \\
& -A_{\zeta(s ; X)} V+(D \zeta)(s ; V ; X),
\end{aligned}
$$

where we have used Gauss and Weingarten equations of the immersion $f: M \rightarrow S(1)$ and the fact that $X, \xi(s ; X)$ and $\zeta(s ; X)$ are orthogonal to $V$. Q.E.D.

REMARK. For arbitrary $V \in T_{X} M$ (not necessarily unit vector) orthogonal to $X$, we also define $\xi_{X}(s ; V), \zeta_{X}(s ; V),(D \xi)(s ; V ; X)$ and $(D \zeta)(s ; V ; X)$ in a trivial manner. Thus in the above theorem, the tangent vector $V$ in the initial conditions need not be a unit vector, because both sides of equations (2.1) and (2.2) are linear with respect to $V$.

COROllaRY 2.2. Let $V$ be an arbitrary tangent vector orthogonal to a unit vector $X$. Then for any $s \in \mathbf{R}$ we have

$$
\begin{aligned}
f_{1}(s)(D \xi) & (s ; V ; X) \\
= & -\xi_{X}\left(s ; A_{\zeta(s ; X)} V\right)+F(s) \zeta_{X}(s ; V)-\zeta_{X}\left(s ; A_{\xi(s ; X)} V\right), \\
\left(f_{1}(s)\right)^{2} H(V, X)+f_{1}(s)(D \zeta)(s ; V ; X) & \\
= & F(s) \xi_{X}(s ; V)-\xi_{X}\left(s ; A_{\xi(s ; X)} V\right)-\zeta_{X}\left(s ; A_{\zeta(s ; X)} V\right) .
\end{aligned}
$$

Proof. Let $\gamma$ be a unit speed geodesic such that $\gamma(0)=x$ and $\dot{\gamma}(0)=X$. Since $\left\{J_{V}(s) ; V \in\{X\}^{\perp}\right\}$ spans the subspace $\{\dot{\gamma}\}^{\perp}$ in $T_{\gamma} M$, then if $\gamma(s)$ is not a conjugate point of $x$, there is a tangent vector $W \in\{X\}^{\perp}$ such that $J_{V}^{*}(s)=J_{W}(s)$ at every nonconjugate point $\gamma(s)$ of $x$, where we note that $W$ may depend on $s$. Thus we have from (2.1) and (2.2)

$$
\begin{gathered}
f_{1}(s) W=F(s) V-A_{\xi(s ; X)} V-A_{\zeta(s ; X)} V \\
\xi_{X}(s ; W)+\zeta_{X}(s ; W)=f_{1}(s) H(V, X)+(D \xi)(s ; V ; X)+(D \zeta)(s ; V ; X) .
\end{gathered}
$$

Substituting the first equation into the second one, we have

$$
\begin{gathered}
f_{1}(s)\left[f_{1}(s) H(V, X)+(D \xi)(s ; V ; X)+(D \zeta)(s ; V ; X)\right] \\
=\xi_{X}\left(s ; F(s) V-A_{\xi(s ; X)} V-A_{\zeta(s ; X)} V\right) \\
+\zeta_{X}\left(s ; F(s) V-A_{\xi(s ; X)} V-A_{\zeta(s ; X)} V\right) .
\end{gathered}
$$


Since both sides of this equation are continuous with respect to $s$, we see that this equation is valid for every $s \in \mathbf{R}$. Exchange $X$ for $-X$. Then we have

$$
\begin{gathered}
f_{1}(s)\left[-f_{1}(s) H(V, X)+(D \xi)(s ; V ; X)-(D \zeta)(s ; V ; X)\right] \\
=-\xi_{X}\left(s ; F(s) V-A_{\xi(s ; X)} V+A_{\zeta(s ; X)} V\right) \\
+\zeta_{X}\left(s ; F(s) V-A_{\xi(s ; X)} V+A_{\zeta(s ; X)} V\right) .
\end{gathered}
$$

Therefore, we obtain the desired equations. Q.E.D.

3. Blaschke structure and the second fundamental form. In the sequel, we shall assume that $M$ is compact. Hence by Theorem 1.3 we know $M$ is a Blaschke manifold. Here we recall the definition of a Blaschke manifold (cf. [2]). Let $N$ be a Riemannian manifold. Let $x \in N$ and $\operatorname{Cut}(x)$ be the cut-locus of $x$ in $N$. If for every $y \in \operatorname{Cut}(x)$ the link $\left\{\dot{\gamma}(y) \in U_{y} N: \gamma\right.$ is a minimal geodesic from $x$ to $\left.y\right\}$ is a great sphere of $U_{y} N$, then $N$ is called a Blaschke manifold at the point $x$. Moreover, $N$ is said to be a Blaschke manifold if it is a Blaschke manifold at every point in $N$. It is well known that $N$ is a Blaschke manifold at $x$ if and only if the distance from $x$ to each point of $\operatorname{Cut}(x)$ is constant (cf. [2, Proposition 5.44, p. 138]). If $N$ is a Blaschke manifold, then every geodesic is a simply closed geodesic loop with the same period (cf. [2, Proposition 5.39, p. 136]). We shall denote the length of geodesics of $M$ by $2 L$. Let $X \in U_{x} M$ and $y=\exp _{x} L X \in \operatorname{Cut}(x)$. Then define $\mathscr{H}_{x}(X)$ by

$$
\mathscr{H}_{x}(X)=\operatorname{Span}\{\dot{\gamma}(0): \gamma \text { is a minimal geodesic from } x \text { to } y\} \text {. }
$$

This subspace is orthogonal to the tangent space $T_{x} \operatorname{Cut}(y)$ of $\operatorname{Cut}(y)$ at $x$ (cf. [2, Proposition 5.39, p. 136]). The dimension of $\mathscr{H}_{x}(X) \cap U_{x} M$ is constant for every $x \in M$ and $X \in U_{x} M$, which is equal to the index of the first conjugate point $y$ of $x$ along a geodesic from $x$ to $y$. Let $e=\operatorname{dim} \mathscr{H}_{x}(X)$. Then by [2, Theorem 7.23, p. 186] we know that $e$ is equal to $1,2,4,8$, or $n$, corresponding to which $n=m, 2 m, 4 m$, 16 , or any $(m=1,2, \ldots)$. We notice that if $M$ is a complex projective space with the canonical metric, then $\mathscr{H}_{x}(X)$ is the holomorphic section determined by $X$. Let $\mathscr{H}_{x}^{*}(X)$ be the orthogonal complement of $X$ in $\mathscr{H}_{x}(X)$. We have

LEMMA 3.1. The subspaces $\mathscr{H}_{x}(X)$ and $\mathscr{H}_{x}^{*}(X)$ are given by

$$
\begin{aligned}
& \mathscr{H}_{x}(X)=\operatorname{Span}\left\{Z \in U_{x} M: \xi(X)=\xi(Z)\right\}, \\
& \mathscr{H}_{x}^{*}(X)=\left\{Z \in T_{x} M: \xi_{X}(Z)=0, Z \perp X\right\},
\end{aligned}
$$

where $\xi(X)=\xi(L ; X)$ and $\xi_{X}(Z)=\xi_{X}(L ; Z)$.

Proof. Note that $f_{j}\left(1 \leqslant j \leqslant d^{*}\right)$ is a periodic function with period $2 L$. If $j$ is an odd integer, then $f_{j}$ is an odd function. Thus we have $f_{j}(L)=0$ for every odd integer $j\left(1 \leqslant j<d^{*}\right)$. It follows that $\zeta(L ; X)=0$. Therefore using Theorem 1.2 , the cut-locus $\operatorname{Cut}(x)$ of $x$ is given by $\operatorname{Cut}(x)=\left\{y: y=F(L) x+\xi(X), X \in U_{x} M\right\}$. We see that $\exp _{x} L X=\exp _{x} L Z$ if and only if $\xi(X)=\xi(Z)$, which shows the first equation. It is clear that $\mathscr{H}_{x}^{*}(X) \subset\left\{Z \in T_{x} M: \xi_{X}(Z)=0, Z \perp X\right\}$. For $Z \in T_{x} M$ such that $Z \perp X, \xi_{X}(Z)=0$ if and only if $J_{Z}(L)=0$ because of (2.1). Since the index of the first conjugate point $\exp _{x} L X$ of $x$ is equal to $e-1$, we have

$$
\operatorname{dim} \mathscr{H}_{x}^{*}(X)=\operatorname{dim}\left\{Z \in T_{x} M: \xi_{X}(Z)=0, Z \perp X\right\} \text {. Q.E.D. }
$$


REMARK. If $Z \in \mathscr{H}_{x}(X) \cap U_{x} M$, then $\mathscr{H}_{x}(X)=\mathscr{H}_{x}(Z)$.

Let $\gamma$ be a unit speed geodesic such that $\gamma(0)=x$ and $\dot{\gamma}(0)=X$. Define $(D \xi)(X)$ by

$$
(D \xi)(X)=\sum f_{j}(L) D \tilde{\tau}^{(j)}(X),
$$

where $D \tilde{\tau}^{(j)}(X)=\nabla_{X}^{\perp}\left(\tilde{\tau}^{(j)}(\dot{\gamma})\right), \nabla^{\perp}$ being the normal connection of the embed$\operatorname{ding} f: M \rightarrow S(1)$.

Lemma 3.2. Let $y=\gamma(L)$. Then

$$
\begin{gathered}
\mathscr{H}_{y}(\dot{\gamma}(L))=\operatorname{Span}\left\{a Z+(D \xi)(Z): Z \in \mathscr{H}_{x}(X),\|Z\|=1\right\}, \\
T_{y} \operatorname{Cut}(x)=\left\{\xi_{X}(Y): Y \in T_{x} \operatorname{Cut}(y)\right\},
\end{gathered}
$$

where $a=f_{1}^{\prime}(L)$.

Proof. Since $\mathscr{H}_{y}(\dot{\gamma}(L))=\operatorname{Span}\{\dot{\alpha}(L): \alpha$ is a unit speed geodesic from $x$ to $y\}$, for the first equality we have only to show $\dot{\alpha}(L)=a Z+(D \xi)(Z)$ if $\dot{\alpha}(0)=Z$. Since $\alpha(s+L)=b \alpha(s)+\xi(\dot{\alpha}(s))$, where $b=F(L)$, we have

$$
\dot{\alpha}(L)=b Z-A_{\xi(Z)}(Z)+(D \xi)(Z) .
$$

On the other hand, from Theorem $1.2, \dot{\alpha}(L)=a Z+\zeta^{\prime}(L ; Z)$, where $\zeta^{\prime}(L ; Z)=$ $\sum f_{j}^{\prime}(L) \tau^{(j)}(Z)$. Hence $b Z-A_{\xi(Z)} Z=a Z$ and $(D \xi)(Z)=\zeta^{\prime}(L ; Z)$. Next we prove the second equality. Consider the linear map $\xi_{X}: V \in\{X\}^{\perp} \mapsto \xi_{X}(V)$. The curve $\theta \mapsto b x+\xi(\cos \theta X+\sin \theta V)$ is contained in $\operatorname{Cut}(x)$. Thus $\xi_{X}(V) \in T_{y} \operatorname{Cut}(x)$. Since $\operatorname{Ker} \xi_{X}=\mathscr{H}_{x}^{*}(X)$ and $\operatorname{dim} T_{x} \operatorname{Cut}(y)=\operatorname{dim} T_{y} \operatorname{Cut}(x)$, we see that $\xi_{X}$ induces an isomorphism from $T_{x} \operatorname{Cut}(y)$ to $T_{y} \operatorname{Cut}(x)$. Q.E.D.

In the proof of the above lemma, we have also proved

$$
A_{\xi(X)} X=(b-a) X
$$

for every $X \in U_{x} M$. Furthermore, we note that $J_{Y}(L)=\xi_{X}(Y)$ for $Y \in T_{x} \operatorname{Cut}(y)$.

Hereafter we shall assume that the helical embedding $f: M \rightarrow S(1)$ is minimal. Thus by Theorem 1.5, $M$ is a globally harmonic manifold and, moreover, if $M$ is simply connected, then $M$ is a strongly harmonic manifold.

LEMMA 3.3. We have $a \neq 0$, where $a=f_{1}^{\prime}(L)$.

Proof. Let $X \in T_{x} M-\{0\}$ and let $\left\{V_{i}\right\}, i=2, \ldots, n$, be an orthonormal base in $\{X\}^{\perp}$.

Let $\left\{J_{i}\right\}, i=2, \ldots, n$, be Jacobi fields along the geodesic

$$
\gamma: s \mapsto \exp _{x} \frac{s}{\|X\|} X
$$

such that $J_{i}(0)=0$ and $J_{i}^{\prime}(0)=V_{i}$ for every $i$. We define $\theta: T M \rightarrow \mathbf{R}$ by

$$
\theta(0)=1, \quad \theta(X)=\|X\|^{-n+1} \operatorname{det}\left(J_{2}(\|X\|), \ldots, J_{n}(\|X\|)\right),
$$

where the determinant should be understood with respect to the parallel frame field along $\gamma$ such that it is equal to $\left\{V_{i}\right\}$ at $s=0$. Since $M$ is globally harmonic, there exists a $C^{\infty}$-function $\Theta: \mathbf{R}_{+} \rightarrow \mathbf{R}$ such that $\theta(X)=\Theta(\|X\|)$. Consider the equation (6.2) in [8, p. 78];

$$
f_{1}^{\prime}+f_{1} \cdot\left((n-1) / s+\Theta^{\prime} / \Theta\right)=n F
$$


Assume that $a=f_{1}^{\prime}(L)=0$. Since $f_{1}(L)=f_{1}^{\prime \prime}(L)=0$ and, from the definition, $F^{\prime}=-f_{1}$, we have

$$
F(s)=b+b_{4}(s-L)^{4}+O\left[(s-L)^{6}\right],
$$

where $b_{4}=F^{(4)}(L) / 4$ !. In [1, p. 110] Allamigeon proved

$$
\Theta(s)=A(s-L)^{e-1}+O\left[(s-L)^{e}\right], \quad(A \neq 0) .
$$

It follows that

$$
\Theta^{\prime} / \Theta=(e-1)(s-L)^{-1}+O(1)
$$

Thus, from (3.2) we have

$$
\begin{aligned}
& -12 b_{4}(s-L)^{2}+O\left[(s-L)^{4}\right] \\
& -\left\{4 b_{4}(s-L)^{3}+O\left[(s-L)^{5}\right]\right\}\left\{(e-1)(s-L)^{-1}+O(1)\right\} \\
& =n\left\{b+b_{4}(s-L)^{4}+O\left[(s-L)^{6}\right]\right\}
\end{aligned}
$$

on some interval $(L-\varepsilon, L], \varepsilon>0$. Thus $b=b_{4}=0$. Repeating this argument, we see that all derivatives of $F$ at $s=L$ vanish. Since $F$ is analytic, we conclude $F \equiv 0$, which contradicts $F(0)=1$. Q.E.D.

Now we characterize $\mathscr{H}_{x}(X)$ and $T_{x} \operatorname{Cut}(y)\left(y=\exp _{x} L X\right)$ as eigenspaces of the second fundamental tensor $A_{\xi(X)}$.

THEOREM 3.4. The subspace $\mathscr{H}_{x}(X)$ and the tangent space of $\operatorname{Cut}(y)$ at $x$ are characterized by

$$
\begin{gathered}
\mathscr{H}_{x}(X)=\left\{Z \in T_{x} M: A_{\xi(X)} Z=(b-a) Z\right\}, \\
T_{x} \operatorname{Cut}(y)=\left\{Y \in T_{x} M: A_{\xi(X)} Y=b Y\right\} .
\end{gathered}
$$

Proof. Let $Z \in \mathscr{H}_{x}(X) \cap U_{x} M$. By Lemma 3.1 and (3.1) we have $A_{\xi(X)} Z=$ $A_{\xi(Z)} Z=(b-a) Z$. Thus $\mathscr{H}_{x}(X)$ is contained in the eigenspace of $A_{\xi(X)}$ corresponding to the eigenvalue $b-a$. Since $T_{x} M=\mathscr{H}_{x}(X) \oplus T_{x} \operatorname{Cut}(y)$ (orthogonal direct sum), it suffices to show that $T_{x} \operatorname{Cut}(y)$ is contained in the eigenspace corresponding to the eigenvalue $b$ of $A_{\xi(X)}$. Let $Y \in T_{x} \operatorname{Cut}(y)$ and let $\gamma$ be the unit speed geodesic such that $\gamma(0)=x$ and $\dot{\gamma}(0)=X$. From Lemma 3.2, we see that $Y \mapsto J_{Y}(L)=\xi_{X}(Y)$ gives a linear isomorphism from $T_{x} \operatorname{Cut}(y)$ to $T_{y} \operatorname{Cut}(x)$. Exchanging $x$ for $y$, we have a Jacobi field $K_{\tilde{Y}}$ along $\gamma$ such that $K_{\tilde{Y}}(L)=0$, $\nabla_{\dot{\gamma}} K_{\tilde{Y}}(L)=\tilde{Y} \in T_{y} \operatorname{Cut}(x)$ and $K_{\tilde{Y}}(0)=Y$. If we put $W=\nabla_{X} K_{\tilde{Y}}(0)$, then $K_{\tilde{Y}}=$ $J_{Y}^{*}+J_{W}$. By Theorem 2.1 we have

$$
K_{\tilde{Y}}(L)=b Y-A_{\xi(X)} Y+(D \xi)(Y ; X)+\xi_{X}(W),
$$

where $(D \xi)(Y ; X)=(D \xi)(L ; Y ; X)$. Thus $A_{\xi(X)} Y=b Y$. Q.E.D.

COROLlary 3.5. We have the equality $a=m b(m=n / e)$.

Proof. Since $f: M \rightarrow S(1)$ is minimal, trace $A_{\xi(X)}=0$. Hence $e(b-a)+$ $(n-e) b=0$. Q.E.D. 
We will now consider $W$ in the proof of Theorem 3.4. Since $\nabla_{\dot{\gamma}} K_{\tilde{Y}}(L)=\tilde{Y}$, we have

$$
\begin{aligned}
\left.\frac{d}{d s} K_{\tilde{Y}}\right|_{s=L} & =\left.\left[\bar{\nabla}_{\dot{\gamma}} K_{\tilde{Y}}+\left\langle\frac{d}{d s} K_{\tilde{Y}}, \gamma(s)\right\rangle \gamma(s)\right]\right|_{s=L} \\
& =\nabla_{\dot{\gamma}} K_{\tilde{Y}}(L)+H\left(\dot{\gamma}(L), K_{\tilde{Y}}(L)\right) \\
& =\tilde{Y} \in T_{y} \operatorname{Cut}(x),
\end{aligned}
$$

where $\bar{\nabla}$ denotes the covariant differentiation on $S(1)$. On the other hand, Theorem 2.1 implies

$$
\begin{aligned}
\left.\frac{d}{d s}\left(J_{Y}^{*}+J_{W}\right)\right|_{s=L}= & -A_{(D \xi)(X)} Y+a H(X, Y) \\
& +\left(D^{2} \xi\right)(Y, X, \ldots, X)+a W+(D \xi)_{X}(W),
\end{aligned}
$$

where we have defined $\left(D^{2} \xi\right)(Y, X, \ldots, X)$ and $(D \xi)_{X}(W)$ by

$$
\begin{aligned}
& \left(D^{2} \xi\right)(Y, X, \ldots, X)=\left(D \zeta^{\prime}\right)(L ; Y ; X)=\left.\frac{d}{d s}(D \zeta)(s ; Y ; X)\right|_{s=L}, \\
& (D \xi)_{X}(W)=\zeta_{X}^{\prime}(L ; W)=\left.\frac{d}{d s} \zeta_{X}(s ; W)\right|_{s=L} .
\end{aligned}
$$

Therefore, by Lemma 3.2 we have $W=\frac{1}{a} A_{(D \xi)(X)} Y$ and

$$
\tilde{Y}=a H(X, Y)+\left(D^{2} \xi\right)(Y, X, \ldots, X)+\frac{1}{a}(D \xi)_{X}\left(A_{(D \xi)(X)} Y\right) .
$$

Moreover, we obtain

$$
(D \xi)(Y ; X)=-\frac{1}{a} \xi_{X}\left(A_{(D \xi)(X)} Y\right)
$$

for every $Y \in T_{x} \operatorname{Cut}(y)$.

Proposition 3.6. If $Y \in T_{x} \operatorname{Cut}(y)$ and $Z \in \mathscr{H}_{x}^{*}(X)$, then

$$
J_{Y}^{*}(L)=-\frac{1}{a} \xi_{X}\left(A_{(D \xi)(X)} Y\right) \in T_{y} \operatorname{Cut}(x)
$$

and

$$
J_{Z}^{*}(L)=a Z+(D \xi)_{X}(Z)-\frac{1}{a} \xi_{X}\left(A_{(D \xi)(X)} Z\right) .
$$

Proof. The first equation is clear because of (3.4) and Theorem 2.1. (2.2) shows that $J_{Z}^{*}(L)=a Z+(D \xi)(Z ; X)$. If we differentiate (2.3) with respect to $s$ at $s=L$, then we have

$$
\begin{aligned}
a(D \xi)(Z ; X) & =-\xi_{X}\left(A_{(D \xi)(X)} Z\right)+b(D \xi)_{X}(Z)-(b-a)(D \xi)_{X}(Z) \\
& =a(D \xi)_{X}(Z)-\xi_{X}\left(A_{(D \xi)(X)} Z\right) \text {. Q.E.D. }
\end{aligned}
$$

From Lemma 3.2 we see that $a Z+(D \xi)_{X}(Z) \in \mathscr{H}_{y}^{*}(\dot{\gamma}(L))$ for $Z \in \mathscr{H}_{x}^{*}(X) \cap$ $U_{x} M$. Hence $a Z+(D \xi)_{X}(Z)$ is proportional to $a W+(D \xi)(W)$ for some $W \in$ $\mathscr{H}_{x}(X) \cap U_{x} M$. Thus $Z=W$ and hence we obtain

$$
(D \xi)(Z)=(D \xi)_{X}(Z)
$$

for every $Z \in \mathscr{H}_{x}^{*}(X) \cap U_{x} M$. It follows that the $\mathscr{H}_{y}^{*}(\dot{\gamma}(L))$-component of $J_{Z}^{*}(L)$ is equal to $a Z+(D \xi)(Z)$ and its $T_{y} \operatorname{Cut}(x)$-component is equal to $-\frac{1}{a} \xi_{X}\left(A_{(D \xi)(X)} Z\right)$. 
We notice that $a Z+(D \xi)(Z)$ is the unit tangent vector at $s=L$ of the geodesic $s \mapsto \exp _{x} s Z$. The next proposition asserts that the linear map $Z \mapsto a Z+(D \xi)_{X}(Z)$ from $\mathscr{H}_{x}^{*}(X)$ to $\mathscr{H}_{y}^{*}(\dot{\gamma}(L))$ is an isometry.

Proposition 3.7. If $Z_{1}, Z_{2} \in \mathscr{H}_{x}(X) \cap U_{x} M$, then

$$
\left\langle a Z_{1}+(D \xi)\left(Z_{1}\right), a Z_{2}+(D \xi)\left(Z_{2}\right)\right\rangle=\left\langle Z_{1}, Z_{2}\right\rangle \text {. }
$$

Proof. Taking a unit vector $Z$ orthogonal to $Z_{1}$, we write $Z_{2}=\cos \theta Z_{1}+\sin \theta Z$. Define $\phi(\theta)$ by

$$
\begin{aligned}
\phi(\theta) & =\left\langle a Z_{1}+(D \xi)\left(Z_{1}\right), a Z_{2}+(D \xi)\left(Z_{2}\right)\right\rangle \\
& =a^{2} \cos \theta+\left\langle(D \xi)\left(Z_{1}\right),(D \xi)\left(\cos \theta Z_{1}+\sin \theta Z\right)\right\rangle .
\end{aligned}
$$

Then $\phi^{\prime}(\theta)=-a^{2} \sin \theta+\left\langle(D \xi)\left(Z_{1}\right),(D \xi)\left(-\sin \theta Z_{1}+\cos \theta Z\right)\right\rangle$ where we have used (3.5). Thus we have $\phi^{\prime \prime}(\theta)=-\phi(\theta)$ because $Z_{2}$ is orthogonal to $-\sin \theta Z_{1}+$ $\cos \theta Z$. Since $\phi(0)=1$, it suffices to show $\phi^{\prime}(0)=0$. Since $\|(D \xi)(V)\|^{2}=1-a^{2}$ for every $V \in \mathscr{H}_{x}(X) \cap U_{x} M$, we have $\phi^{\prime}(0)=\left\langle(D \xi)(Z),(D \xi)_{Z}\left(Z_{1}\right)\right\rangle=0$. Q.E.D.

4. Some results. At first we consider the case $d=3$. Since a helical immersion of order 3 is a proper cubic geodesic immersion, we can apply the result obtained by Nakagawa [6] to this case. However, we make use of results in the preceding section.

THEOREM 4.1 [6]. If $f: M \rightarrow S(1)$ is a helical minimal embedding of order 3 of a compact Riemannian manifold $M$ into a unit sphere $S(1)$, then $M$ is isometric to a sphere of constant curvature and $f$ is equivalent to a standard minimal embedding of the sphere ( for the definition of standard minimal immersions, see [2 or 12]).

Proof. Since $f$ is of order 3 and $(D \xi)(X)=\zeta^{\prime}(L ; X)$, we have $(D \xi)(X)=$ $a_{3}^{\prime}\left(\lambda_{1} \lambda_{2}\right)^{-1}(D H)\left(X^{3}\right)$ where $a_{3}^{\prime}=f_{3}^{\prime}(L)$ and we note that $b_{33}=1$ in Theorem 1.1. Assume that $e \geqslant 2$. By virture of the Codazzi equation we see that $D \xi$ satisfies

$$
(D \xi)(U, V, W)=(D \xi)(V, U, W)=(D \xi)(U, W, V)
$$

for every $U, V, W \in T_{x} M$. Thus $(D \xi)(Z ; X)=(D \xi)(Z, X, X)=\frac{1}{3}(D \xi)(Z)$ for $Z \in \mathscr{H}_{x}^{*}(X) \cap U_{x} M$. By Proposition 3.6 and (3.5), we find $\frac{2}{3}(D \xi)(Z)$ $=\frac{1}{a} \xi_{X}\left(A_{(D \xi)(X)} Z\right)$. Since we see from Lemma 3.2 that both sides of this equation are orthogonal, we conclude that $(D \xi)(Z)=0$ and hence $a_{3}^{\prime}=0$. The constants $a, b$ and $a_{3}^{\prime}$ satisfy $a=1-\lambda_{1} f_{2}(L), a_{3}^{\prime}=\lambda_{2} f_{2}(L)-\lambda_{3} f_{4}(L)$ and $b=1-c_{2} f_{2}(L)-$ $c_{4} f_{4}(L)$. Moreover, $c_{2}^{2}+c_{4}^{2}=1$ by [8, Remark, p. 74]. From these equations we obtain $a=b$. By virtue of Corollary 3.5 we have $e=n$. Next assume that $e=1$. Let $Y \in T_{x} \operatorname{Cut}(y)=\{X\}^{\perp}$ such that $\|Y\|=1$. By (3.4), we have $\frac{1}{3}(D \xi)_{X}(Y)=$ $-\frac{1}{a} \xi_{X}\left(A_{(D \xi)(X)} Y\right)$. Noting that $\langle(D \xi)(V), H(V, W)\rangle=0$ for every $V, W \in U_{x} M$ (cf. [8, Lemma 3.3, p. 68]), we find

$$
\langle(D \xi)(X), H(Y, Y)\rangle=-\left\langle(D \xi)_{X}(Y), H(X, Y)\right\rangle \text {. }
$$

Thus,

$$
\frac{1}{3}\langle(D \xi)(X), H(Y, Y)\rangle=\frac{1}{a}\left\langle\xi_{X}\left(A_{(D \xi)(X)} Y\right), H(X, Y)\right\rangle .
$$


Since $\langle\xi(V), H(V, W)\rangle=(b-a)\langle V, W\rangle$ for every $V, W \in U_{x} M$ (cf. [8, Lemma 3.3 , p. 68]), we see that

$$
\begin{aligned}
\frac{1}{3}\langle(D \xi)(X) & H(Y, Y)\rangle \\
= & \frac{1}{a}\left[-\left\langle\xi(X), H\left(A_{(D \xi)(X)} Y, Y\right)\right\rangle+(b-a)\left\langle A_{(D \xi)(X)} Y, Y\right\rangle\right] \\
= & -\langle(D \xi)(X), H(Y, Y)\rangle,
\end{aligned}
$$

where the last equality is derived from Theorem 3.4. It follows that $A_{(D \xi)(X)}=0$ and hence $(D \xi)_{X}(Y)=0$ for every $Y \in\{X\}^{\perp} \cap U_{x} M$. This means that $(D \xi)(V)$ is a constant vector on $U_{x} M$. Since the covariant degree of $D \xi$ is equal to 3 , we have $(D \xi)(X)=0$, and so $a_{3}^{\prime}=0$. We conclude $e=n$ in the same way as the case $e \geqslant 2$. This conclusion contradicts to the assumption $e=1$. In this way we have proved $e=n$. So we can use [2, D.3, Corollary, p. 236 and 5.57, p. 142]. We conclude that $M$ is isometric to a sphere of constant curvature. The second assertion is derived from the fact that the degree of a helical immersion $M \rightarrow S(1)$ is smaller than the order (cf. [8, Proposition 5.6]) and Wallach's linear rigidity theorem (cf. [12, p. 32]). Q.E.D.

THEOREM 4.2. Let $f: M \rightarrow S(1)$ be a minimal helical embedding. If $M$ is compact and $e=n$ (hence $M$ is isometric to a sphere), then $d$ must be an odd integer. In this case, we have $\xi(X)=0$ for every $X \in U_{x} M$ and $a=b=-1$.

Proof. In [8, Theorem 6.7] we have proved

$$
\begin{aligned}
\Delta_{s}(\xi(s ; X)+\zeta(s ; X))= & \xi^{\prime \prime}(s ; X)+\zeta^{\prime \prime}(s ; X) \\
& +\omega(s)\left(\xi^{\prime}(s ; X)+\zeta^{\prime}(s ; X)\right)+n(\xi(s ; X)+\zeta(s ; X)),
\end{aligned}
$$

where $\omega=\left(n F-f_{1}^{\prime}\right) / f_{1}$ and $\Delta_{s}$ denotes the Laplace operator with respect to the metric $g_{s}$ on $U_{x} M$ induced by the map $X \mapsto \exp _{x} s X$ from the induced metric on the geodesic sphere with center $x$ and radius $s$. Since the volume form of the metric $g_{s}$ is equal to $s^{n-1} \theta d v$ where $d v$ is the canonical volume form on $U_{x} M$ (cf., [2, p. 159]), we have from Green's theorem

$$
\int_{U_{x} M}\left[\xi^{\prime \prime}(s ; X)+\omega(s) \xi^{\prime}(s ; X)+n \xi(s ; X)\right] d v=0
$$

for every $s \in(0, L)$. It follows that

$$
\sum\left(f_{j}^{\prime \prime}+\omega f_{j}^{\prime}+n f_{j}\right) \int_{U_{x} M} \tilde{\tau}^{(j)}(X) d v=0 .
$$

From the definition of $f_{j}$ we have

$$
j ! f_{j}(s)=\left(\lambda_{1} \cdots \lambda_{j-1}\right) s^{j}+O\left(s^{j+1}\right), \quad \omega(s)=(n-1) / s+O(s) .
$$

Therefore we obtain

$$
f_{j}^{\prime \prime}+\omega f_{j}^{\prime}+n f_{j}=\frac{1}{(j-1) !}\left(\lambda_{1} \cdots \lambda_{j-1}\right)(n+j-2) s^{j-2}+O\left(s^{j}\right) .
$$

It follows that we have, inductively, $\int_{U_{x} M} \tilde{\tau}^{(j)}(X) d v=0$, from which $\int_{U_{x} M} \xi(X) d v$ $=0$. Since $e=n, \xi(X)$ is constant on $U_{x} M$. Thus we conclude that $\xi(X)=0$. 
Noting that $\|\xi(X)\|^{2}=1-b^{2}$ and $f$ is an embedding, we have $b=-1$. If $d$ is even, then $H(X, X),\left(D^{2} H\right)\left(X^{4}\right), \ldots,\left(D^{d-2} H\right)\left(X^{d}\right)$ are linearly independent and hence $\tilde{\tau}^{(j)}, j=2,4, \ldots, d$, are also linearly independent. Thus $f_{j}(L)=0$ for $j=2,4, \ldots, d$, which implies that $b=1$. This contradicts the assumption that $f$ is an embedding. Q.E.D.

REMARK. The equality $b=-1$ means that the cut-locus $\operatorname{Cut}(x)$ is the antipodal point of $x$ in $S(1)$.

When $d$ is an odd integer, can one show $e=n$ ? In this case we have $\sum c_{j} \tilde{\tau}^{(j)}(X)=0$ (cf. [8, Remark, p. 74]), which is equivalent to $\int_{0}^{L} \xi(s ; X) d s=0$. I do not know other conditions equivalent to $d$ is odd.

Next we shall compute the second fundamental form of $\operatorname{Cut}(y)$ in $M$. Let $\gamma$ be a unit speed geodesic in $M$ such that $\gamma(0)=x, \dot{\gamma}(0)=X$ and $\gamma(L)=y$.

Proposition 4.3. Let $Z \in \mathscr{H}_{x}(X) \cap U_{x} M$ (unit normal vector at $x$ of $\operatorname{Cut}(y)$ in $M)$. Then the second fundamental tensor $\tilde{A}_{Z}$ corresponding to $Z$ of the submanifold $\operatorname{Cut}(y)$ in $M$ is given by

$$
\left\langle\tilde{A}_{Z} Y_{1}, Y_{2}\right\rangle=-\frac{1}{a}\left\langle A_{(D \xi)(Z)} Y_{1}, Y_{2}\right\rangle
$$

for $Y_{1}, Y_{2} \in T_{x} \operatorname{Cut}(y)$. In particular, $\operatorname{Cut}(y)$ is a minimal submanifold in $M$.

Proof. We may assume that $Z=X$ because of $\mathscr{H}_{x}(X)=\mathscr{H}_{x}(Z)$. Consider the Jacobi field $K_{\tilde{Y}}$ as in the proof of Theorem 3.4. Since this Jacobi field is a variation vector of a geodesic variation such that each geodesic is orthogonal to $\operatorname{Cut}(y)$ and passes through $y$ at $s=L$, we have

$$
-\tilde{A}_{X} Y-\nabla_{X} K_{\tilde{Y}}(0) \in \mathscr{H}_{x}(X)
$$

(cf. [4]). Since $\nabla_{X} K_{\tilde{Y}}(0)=W=\frac{1}{a} A_{(D \xi)(X)} Y$, we have

$$
\left\langle\tilde{A}_{X} Y_{1}, Y_{2}\right\rangle=-\frac{1}{a}\left\langle A_{(D \xi)(X)} Y_{1}, Y_{2}\right\rangle \text {. Q.E.D. }
$$

The second fundamental tensor $A_{(D \xi)(X)}$ gives good information for the study of Riemannian structure on $M$. Indeed, we have

Proposition 4.4. The matrix representation of $A_{(D \xi)(X)}$ is given by

$$
\left.\frac{1}{a} A_{(D \xi)(X)}=\left(\begin{array}{c:c:c}
0 & 0 & 0 \\
\hdashline 0 & 0 & Q_{X} \\
\hdashline 0 & t_{Q_{X}} & -\tilde{\tilde{A}}_{X}^{-}
\end{array}\right) \begin{array}{c}
X \\
\} \mathscr{H}_{x}^{*}(X)
\end{array}\right\} T_{x} \operatorname{Cut}(y)
$$

where $Q_{X}$ is an $(e-1) \times(n-e)$ matrix. Let $Q_{\dot{\gamma}}$ be the field of linear transformation $\mathscr{H}_{\gamma}^{*}(\dot{\gamma}) \rightarrow T_{\gamma} \operatorname{Cut}(\gamma(s+L))$ along $\gamma$. Then $Q_{\dot{\gamma}}$ vanishes along $\gamma$ if and only if $\mathscr{H}_{\gamma}(\dot{\gamma})$ is parallel along $\gamma$.

Proof. Let $Z_{1}, Z_{2} \in \mathscr{H}_{x}^{*}(X) \cap U_{x} M$. Then we have

$$
\left\langle A_{(D \xi)(X)} Z_{1}, Z_{2}\right\rangle=-\left\langle(D \xi)\left(Z_{1}\right), H\left(X, Z_{2}\right)\right\rangle
$$

by (3.5). If $Z_{1}=Z_{2}$, then $\left\langle(D \xi)\left(Z_{1}\right), H\left(X, Z_{2}\right)\right\rangle=0$. If $Z_{1}$ is orthogonal to $Z_{2}$, then

$$
\left\langle(D \xi)\left(Z_{1}\right), H\left(X, Z_{2}\right)\right\rangle=-\left\langle(D \xi)\left(Z_{2}\right), H\left(X, Z_{1}\right)\right\rangle
$$


because of (3.5) where we note that $Z_{2} \in \mathscr{H}_{x}^{*}\left(Z_{1}\right)$. However, $\left\langle A_{(D \xi)(X)} Z_{1}, Z_{2}\right\rangle$ is symmetric with respect to $Z_{1}$ and $Z_{2}$, so that $\left\langle A_{(D \xi)(X)} Z_{1}, Z_{2}\right\rangle=0$. Since $A_{(D \xi)(X)} X$ $=0$, we have the first assertion. Let $s_{0} \in[0, L)$ and let $I$ be some interval containing $s_{0}$. Let $Z^{*}$ be a vector field defined on $\gamma(I)$ such that $Z^{*}(s) \in \mathscr{H}_{\gamma(s)}^{*}(\dot{\gamma}(s))$ for each $s \in I$. Let $W$ be an arbitrary tangent vector at $\gamma\left(s_{0}\right)$ which is orthogonal to $\dot{\gamma}\left(s_{0}\right)$ and $W^{*}$ the parallel vector field defined on $\gamma(I)$ such that $W^{*}\left(s_{0}\right)=W$. Since $A_{\xi(\dot{\gamma})} Z^{*}=(b-a) Z^{*}$, we have

$$
\begin{aligned}
\left\langle(D \xi)(\dot{\gamma}), H\left(Z^{*}, W^{*}\right)\right\rangle+ & \left\langle\xi(\dot{\gamma}),(D H)\left(\dot{\gamma}, Z^{*}, W^{*}\right)\right\rangle \\
& +\left\langle\xi(\dot{\gamma}), H\left(\nabla_{\dot{\gamma}} Z^{*}, W^{*}\right)\right\rangle=(b-a)\left\langle\nabla_{\dot{\gamma}} Z^{*}, W^{*}\right\rangle .
\end{aligned}
$$

Noting that $\langle\xi(V),(D H)(V, V, W)\rangle=0$ for any $V, W \in T_{\gamma} M$ (cf. [8, Lemma 3.3]), we see that

$$
2\left\langle\xi(\dot{\gamma}),(D H)\left(\dot{\gamma}, Z^{*}, W^{*}\right)\right\rangle=-\left\langle\xi_{\dot{\gamma}}\left(Z^{*}\right),(D H)\left(\dot{\gamma}, \dot{\gamma}, W^{*}\right)\right\rangle .
$$

Since $Z^{*} \in \mathscr{H}_{\gamma}^{*}(\dot{\gamma})$, the right-hand side vanishes. So we find

$$
Q_{\dot{\gamma}}\left(Z^{*}\right)+A_{\xi(\dot{\gamma})} \nabla_{\dot{\gamma}} Z^{*}=(b-a) \nabla_{\dot{\gamma}} Z^{*},
$$

which shows $Q_{\dot{\gamma}}$ vanishes at $s=s_{0}$ if and only if $\left.\nabla_{\dot{\gamma}} Z^{*}\right|_{s=s_{0}}$ is contained in $\mathscr{H}_{\gamma\left(s_{0}\right)}^{*}\left(\dot{\gamma}\left(s_{0}\right)\right)$. Q.E.D.

Next we shall consider the submersion $\Pi: U_{x} M \rightarrow \operatorname{Cut}(x)$ defined by $\Pi(X)=$ $\exp _{x} L X(=b x+\xi(X))$ (cf. [2, p. 134]), whose fiber through $X$ is the $(e-1)$ dimensional great sphere $\mathscr{H}_{x}(X) \cap U_{x} M$. The total space $U_{x} M$ and the base space $\operatorname{Cut}(x)$ are equipped with the canonical metric and the metric induced from $M$, respectively. We hope that $\Pi$ becomes a Riemannian submersion when the metric on $U_{x} M$ is changed homothetically. In fact, we have Escobales' theorem [3, Theorem 3.5 , p. 273], which states that if $\Pi: S^{m}(1) \rightarrow B$ is a Riemannian submersion with connected totally geodesic fibers $(1 \leqslant \operatorname{dim}$ fiber $\leqslant m-1)$, then $B$ is isometric to one of $\mathbf{C} P^{n}, \mathbf{Q} P^{n}, S^{2}, S^{4}$ and $S^{8}$ with maximal curvature 4. Indeed, for $n \geqslant 2$ and $B=\mathbf{C} P^{n}, \mathbf{Q} P^{n}$ or $S^{2}, \Pi$ is equivalent to the Hopf fibering. Unfortunately, when $B=S^{4}$ or $S^{8}$, the last assertion is not given in [3] (see [3, Remark, p. 271]). Making use of Escobales' result we have

Proposition 4.5. Let $e \neq n$. Define $\eta(X)$ by $\eta(X)=\xi^{\prime \prime}(L ; X)$ for every $X \in U M$ (unit sphere bundle of $M$ ). If $\mu=\left\langle A_{\eta(X)} Y, Y\right\rangle$ is constant for every $x \in M, X \in U_{x} M$ and $Y \in T_{x} \operatorname{Cut}(y) \cap U_{x} M\left(y=\exp _{x} L X\right)$, then the cut-locus $\operatorname{Cut}(x)$ is isometric to one of $\mathbf{C} P^{m-1}, \mathbf{Q} P^{m-1}, S^{2}, S^{4}, S^{8}$ with standard metric of maximal curvature $2(a+\mu) / a$, or $\mathbf{R} P^{n-1}$ with constant curvature $(a+\mu) / 2 a$.

Proof. Let $Y \in T_{x} \operatorname{Cut}(y)$. Consider the Jacobi field $K_{\tilde{Y}}$ along $\gamma: s \mapsto \exp _{x} s X$ satisfying $K_{\tilde{Y}}(0)=Y, K_{\tilde{Y}}(L)=0$ and $\nabla_{\dot{\gamma}} K_{\tilde{Y}}(L)=\tilde{Y} \in T_{y} \operatorname{Cut}(x)$ as in the proof of Theorem 3.4. Then we have $\nabla_{\dot{\gamma}} K_{\tilde{Y}}(0)=\frac{1}{a} A_{(D \xi)(X)} Y$ and $\tilde{Y}$ is given by (3.3). The second derivative of (2.4) at $s=L$ gives

$$
\begin{aligned}
2 a^{2} H(X, V)+ & 2 a\left(D^{2} \xi\right)(V ; X, \ldots, X) \\
= & -a \xi_{X}(V)-\xi_{X}\left(A_{\eta(X)} V\right)+b \eta_{X}(V) \\
& -\eta_{X}\left(A_{\xi(X)} V\right)-2(D \xi)_{X}\left(A_{(D \xi)(X)} V\right),
\end{aligned}
$$


where $\eta_{X}(V)=\xi_{X}^{\prime \prime}(L ; V)$. If $V=Y$, then from Theorem 3.4 we have

$$
\begin{aligned}
a H(X, Y)+ & \left(D^{2} \xi\right)(Y ; X, \ldots, X) \\
& =-\frac{1}{2} \xi_{X}\left(Y+\frac{1}{a} A_{\eta(X)} Y\right)-\frac{1}{a}(D \xi)_{X}\left(A_{(D \xi)(X)} Y\right) .
\end{aligned}
$$

It follows that $\tilde{Y}=-\frac{1}{2} \xi_{X}\left(Y+\frac{1}{a} A_{\eta(X)} Y\right)$. Let $W \in T_{x} \operatorname{Cut}(y)$. Since $\left\langle K_{\tilde{Y}}, \nabla_{\dot{\gamma}} J_{W}\right\rangle-$ $\left\langle\nabla_{\dot{\gamma}} K_{\tilde{Y}}, J_{W}\right\rangle=$ constant along $\gamma$, we find

$$
\frac{1}{2}\left\langle\xi_{X}\left(Y+\frac{1}{a} A_{\eta(X)} Y\right), \xi_{X}(W)\right\rangle=\langle Y, W\rangle
$$

for every $Y, W \in T_{x} \operatorname{Cut}(y)$. Thus the assumption implies that

$$
\left\langle\xi_{X}(Y), \xi_{X}(W)\right\rangle=2 a\langle Y, W\rangle /(a+\mu)
$$

for every $Y, W \in T_{x} \operatorname{Cut}(y)$. Consider the metric $2 a /(a+\mu)\langle$,$\rangle on U_{x} M$ and note that $T_{x} \operatorname{Cut}(y)$ is the horizontal space at $X \in U_{x} M$ of the submersion $\Pi$. Since

$$
\Pi_{*} Y=\left.\frac{d}{d \theta} \xi(\cos \theta X+\sin \theta Y)\right|_{\theta=0}
$$

for $Y \in T_{x} \operatorname{Cut}(y) \cap U_{x} M$, we see that $\Pi$ is a Riemannian submersion. Therefore if $e=1$, then $\operatorname{Cut}(x)$ is isometric with $\mathbf{R} P^{n-1}$ of constant curvature $(a+\mu) / 2 a$. If $e=2,4$, or 8 , then by Escobales' theorem, $\operatorname{Cut}(x)$ is isometric to one of $\mathbf{C P} P^{m-1}$, $\mathbf{Q} P^{m-1}, S^{2}, S^{4}$, or $S^{8}$ with maximal sectional curvature $2(a+\mu) / a$. Q.E.D.

Note. Under the assumption of Proposition 4.5, can one show $M$ is isometric to one of $\mathbf{R} P^{n}, \mathbf{C} P^{m}, \mathbf{Q} P^{m}$ or $\mathbf{C} a y P^{2}$ with standard metrics? Perhaps this is possible if the cut-loci are totally geodesic submanifolds in $M$. It seems that the fact that $\Pi$ is equivalent to Hopf fibering is useful to show that the cut-loci are totally geodesic in $M$. However, the corresponding homogeneity of the submersion in the case $B=S^{4}$ or $S^{8}$ is not given in [3].

5. Helical immersions of order 4 . In the present and subsequent sections we shall consider the helical minimal embedding of order 4 of compact Riemannian manifold $M$ into a unit sphere $S(1)$. By Theorem 4.2 we have $e \neq n$. For the sake of convenience we shall put

$$
a_{2}=f_{2}(L), \quad a_{4}=f_{4}(L), \quad a_{3}^{\prime}=f_{3}^{\prime}(L) \text { and } r^{2}=c_{2}^{2}+c_{4}^{2} .
$$

By definition we have $b=1-c_{2} a_{2}-c_{4} a_{4}$, and hence for $X \in U_{x} M$

$$
\begin{aligned}
\xi(X) & =a_{2} \lambda_{1}^{-1} H(X, X)+a_{4}\left(\lambda_{1} \lambda_{2} \lambda_{3}\right)^{-1}\left[\lambda_{2}^{2} H(X, X)+\left(D^{2} H\right)\left(X^{4}\right)\right] \\
& =(1-b) H(X, X)+a_{4}\left(\lambda_{1} \lambda_{2} \lambda_{3}\right)^{-1}\left(D^{2} H\right)\left(X^{4}\right),
\end{aligned}
$$

where we note that $b_{42}=\lambda_{2}^{2}$ (cf. Theorem 1.1 and [8, Theorem 4.1]). We also have

$$
(D \xi)(X)=a_{3}^{\prime}\left(\lambda_{1} \lambda_{2}\right)^{-1}(D H)(X, X, X)
$$

for $X \in U_{x} M$. Noting that $s$ is the arc-length parameter and $f\left(\exp _{x} s X\right) \in S(1)$ in Theorem 1.2 , we have $\sum_{i=1}^{4}\left(f_{i}^{\prime}\right)^{2}=1$ and $\sum_{i=1}^{4} f_{i}^{2}=2(1-F)$. Thus we obtain

$$
\begin{gathered}
a^{2}+\left(a_{3}^{\prime}\right)^{2}=1, \\
a_{2}^{2}+a_{4}^{2}=2(1-b) .
\end{gathered}
$$


Since $f_{1}^{\prime}=1-\lambda_{1} f_{2}$ and $f_{3}^{\prime}=\lambda_{2} f_{2}-\lambda_{3} f_{4}$, we have

$$
\begin{gathered}
a_{2}=c_{2}(1-a), \\
a_{4}=c_{4}(1-a)-\lambda_{3}^{-1} a_{3}^{\prime} .
\end{gathered}
$$

Substitute (5.5) and (5.6) into the equation $\left(a_{2}-c_{2}\right)^{2}+\left(a_{4}-c_{4}\right)^{2}=r^{2}$ which is derived from (5.4). Then we find

$$
\left(\lambda_{3}^{-2} a_{3}^{\prime}+2 c_{4} \lambda_{3}^{-1} a\right) a_{3}^{\prime}=r^{2}\left(1-a^{2}\right),
$$

from which we see that $a_{3}^{\prime}=0$ or $a_{3}^{\prime}=2 c_{4} \lambda_{3} a /\left(\lambda_{3}^{2} r^{2}-1\right)$.

First, we shall consider the case (I): $a_{3}^{\prime}=0$. In this case, $a^{2}=1$. If $a=1$, then $a_{2}=0$ and $a_{4}=0$ because of (5.5) and (5.6), respectively. Thus $b=1$. This result contradicts the assumption that $f$ is an embedding. Thus we have $a=-1$. We need the following

Lemma 5.1. Let $f: M \rightarrow S(1)$ be a helical minimal embedding of a compact Riemannian manifold $M$. Then we have

$$
\eta(X)=a H(X, X)+\left(D^{2} \xi\right)(X),
$$

$$
\begin{aligned}
& a H(X, Z)+\left(D^{2} \xi\right)(Z ; X, \ldots, X) \\
&=-\frac{1}{2 a} \xi_{X}\left(A_{\eta(X)} Z\right)+\frac{1}{2} \eta_{X}(Z)-\frac{1}{a}(D \xi)_{X}\left(A_{(D \xi)(X)} Z\right)
\end{aligned}
$$

for $X \in U_{x} M$ and $Z \in \mathscr{H}_{x}^{*}(X)$.

Proof. Let $\gamma$ be a unit speed geodesic such that $\dot{\gamma}(0)=X \in U_{x} M$. Then by Theorem 1.2 we have

$$
\begin{aligned}
\gamma(s+t) & =F(s+t) x+f_{1}(s+t) X+\xi(s+t ; X)+\zeta(s+t ; X) \\
& =F(t) \gamma(s)+f_{1}(t) \dot{\gamma}(s)+\xi(t ; \dot{\gamma}(s))+\zeta(t ; \dot{\gamma}(s)) .
\end{aligned}
$$

Differentiate this equation with respect to $s$ at $s=0$. Then

$$
\begin{aligned}
-f_{1}(t) x+f_{1}^{\prime}(t) X+ & \xi^{\prime}(t ; X)+\zeta^{\prime}(t ; X) \\
= & F(t) X+f_{1}(t)(H(X, X)-x)-A_{\xi(t ; X)} X+(D \xi)(t ; X) \\
& -A_{\zeta(t ; X)} X+(D \zeta)(t ; X),
\end{aligned}
$$

where we have used Gauss and Weingarten equations. We know $A_{\zeta(t ; X)} X=0$ by $[\mathbf{8}$, Lemma 3.3]. It follows that

$$
A_{\xi(t ; X)} x=\left(F(t)-f_{1}^{\prime}(t)\right) X, \xi^{\prime}(t ; X)=f_{1}(t) H(X, X)+(D \zeta)(t ; X)
$$

and

$$
\zeta^{\prime}(t ; X)=(D \xi)(t ; X)
$$

Therefore, we see that

$$
\begin{aligned}
\xi^{\prime \prime}(t ; X) & =f_{1}^{\prime}(t) H(X, X)+\left(D \zeta^{\prime}\right)(t ; X) \\
& =f_{1}^{\prime}(t) H(X, X)+\left(D^{2} \xi\right)(t ; X) .
\end{aligned}
$$

In particular, at $t=L$ we obtain (5.7).

Let $V=Z \in \mathscr{H}_{x}^{*}(X)$ in (4.1). Since $A_{\xi(X)} Z=(b-a) Z$ and $\xi_{X}(Z)=0$, we obtain (5.8). Q.E.D. 
Since $(D \xi)(X)=0$ for every $X \in U M$, we have $\eta(X)=-H(X, X)$ because of (5.7) and hence $\eta_{X}(Z)=-2 H(X, Z)$ for $Z \in \mathscr{H}_{x}^{*}(X)$. Thus (5.8) implies $\xi_{X}\left(A_{\eta(X)} Z\right)=0$ which means that $A_{\eta(X)} \mathscr{H}_{x}^{*}(X) \subset \mathscr{H}_{x}^{*}(X)$. Noting that $A_{\eta(X)} X$ is proportional to $X$ (cf. [8, Lemma 3.3]), we conclude that $A_{\eta(X)} T_{x} \operatorname{Cut}(y) \subset T_{x} \operatorname{Cut}(y)$. Let $Y_{i}, i=e+1, \ldots, n$, be an orthonormal base in $T_{x} \operatorname{Cut}(y)$ such that $A_{\eta(X)} Y_{i}=$ $\mu_{i} Y_{i}$. Then from (4.2) we find

$$
-H\left(X, Y_{i}\right)=-\frac{1}{2}\left(1-\mu_{i}\right) \xi_{X}\left(Y_{i}\right)
$$

for each $i$. It follows that

$$
\begin{aligned}
\mu_{i} & =\left\langle A_{\eta(X)}, Y_{i}, Y_{i}\right\rangle=-\left\langle H(X, X), H\left(Y_{i}, Y_{i}\right)\right\rangle \\
& =2\left\|H\left(X, Y_{i}\right)\right\|^{2}-\left(\lambda_{1}^{2}-1\right) \\
& =\left(1-\mu_{i}\right)\left\langle\xi_{X}\left(Y_{i}\right), H\left(X, Y_{i}\right)\right\rangle-\left(\lambda_{1}^{2}-1\right),
\end{aligned}
$$

where for the third equality we have used the fact that a helical immersion is $\kappa_{1}^{2}$-isotropic, as well as [7, (3.5), p. 40]. Since

$$
\left\langle\xi_{X}\left(Y_{i}\right), H\left(X, Y_{i}\right)\right\rangle=-\left\langle\xi(X), H\left(Y_{i}, Y_{i}\right)\right\rangle+b-a=1,
$$

we obtain $\mu_{i}=\left(2-\lambda_{1}^{2}\right) / 2$ for each $i$. Hence by Propositions 4.3 and 4.5 the cut-locus is a totally geodesic submanifold in $M$ and isometric to one of $\mathbf{C P} P^{m-1}$, $\mathbf{Q} P^{m-1}(m \geqslant 2), S^{2}, S^{4}$ with standard metric of maximal sectional curvature $\lambda_{1}^{2}$, or $\mathbf{R} P^{n-1}$ with constant curvature $\lambda_{1}^{2} / 4$.

Assume $e \geqslant 2$. Let $Y_{0} \in T_{x} \operatorname{Cut}(y) \cap U_{x} M$ and $x_{0}=\exp _{x} L Y_{0}$. Then $\mathscr{H}_{x}\left(Y_{0}\right) \subset$ $T_{x} \operatorname{Cut}(y)$ by virtue of the above conclusion. Thus $\mathscr{H}_{x}(X) \subset T_{x} \operatorname{Cut}\left(x_{0}\right)$. Since the sectional curvature of $\operatorname{Cut}\left(x_{0}\right)$ corresponding to a section in $\mathscr{H}_{x}(X)$ is equal to $\lambda_{1}^{2}$, [7, (3.6), p. 40] implies $H(X, Z)=0$ for every $Z \in \mathscr{H}_{x}^{*}(X)$. Therefore

$$
(D H)(X, X, Z)=0 \text { for } Z \in \mathscr{H}_{x}^{*}(X)
$$

because of Proposition 4.4.

Next we shall prove $(D H)(X, X, Y)=0$ for $Y \in T_{x} \operatorname{Cut}(y)$. Recall the Jacobi field $K_{\tilde{Y}}$ along $\gamma: s \mapsto \exp _{x} s X$ such that $K_{\tilde{Y}}(0)=Y, K_{\tilde{Y}}(L)=0$ and $\nabla_{\dot{\gamma}} K_{\tilde{Y}}(L)=\tilde{Y}$ $\in T_{y} \operatorname{Cut}(x)$, where $\tilde{Y}$ is given by

$$
\tilde{Y}=-\frac{1}{2} \xi_{X}\left(Y+\frac{1}{a} A_{\eta(X)} Y\right) .
$$

It is easy to show $d^{2} K_{\tilde{Y}}(L) / d s^{2}=2 H(\dot{\gamma}(L), \tilde{Y})$. On the other hand, since $K_{\tilde{Y}}=J_{Y}^{*}$ $+J_{A_{(D \xi)(X)} Y / a}$, Theorem 2.1 implies

$$
d^{2} K_{\tilde{Y}}(L) / d s^{2}=-a Y-A_{\eta(X)} Y+(D \eta)(Y ; X)+\eta_{X}\left(\frac{1}{a} A_{(D \xi)(X)} Y\right),
$$

where $(D \eta)(Y ; X)=\left(D \xi^{\prime \prime}\right)(L ; Y ; X)$. Thus we have

$$
(D \eta)(Y ; X)=2 H(\dot{\gamma}(L), \tilde{Y})+a Y+A_{\eta(X)} Y-\eta_{X}\left(\frac{1}{a} A_{(D \xi)(X)} Y\right) .
$$

Computing the third derivative of (2.3) at $s=L$, we have

$$
\begin{aligned}
3 a(D \eta) & (Y ; X)+f_{1}^{\prime \prime \prime}(L)(D \xi)(Y ; X) \\
& =-\xi_{X}\left(A_{(D \eta)(X)} Y\right)-3 \eta_{X}\left(A_{(D \xi)(X)} Y\right)-3 a(D \xi)_{X}\left(Y+\frac{1}{a} A_{\eta(X)} Y\right)
\end{aligned}
$$


(see the proof of Lemma 5.3). Since $(D \xi)(X)=0$ for every $X \in U M$, we see that $(D \eta)(Y ; X) \in T_{y} \operatorname{Cut}(x)$, from which $2 H(\dot{\gamma}(L), \tilde{Y})-Y+\left(1-\lambda_{1}^{2} / 2\right) Y \in$ $T_{y} \operatorname{Cut}(x)$. But this vector is a normal vector at $y$. Thus we conclude $(D H)(Y, X, X)$ $=-(D \eta)(Y ; X)=0$.

In this way, $(D H)(X, X, V)=0$ for every $V \in T_{x} M$ orthogonal to $X$. This shows that $(D H)(X, X, X)$ is a constant vector on $U_{x} M$ and so $D H=0$. This result contradicts the assumption that the order $d=4$.

Secondly we shall consider the case (II): $a_{3}^{\prime} \neq 0$. In this case, we showed

$$
a_{3}^{\prime}=2 c_{4} \lambda_{3} a /\left(\lambda_{3}^{2} r^{2}-1\right)
$$

so by definition of $c_{4}$ and $r^{2}$,

$$
a_{3}^{\prime}=2 \lambda_{1} \lambda_{2} a /\left(\lambda_{2}^{2}+\lambda_{3}^{2}-\lambda_{1}^{2}\right) .
$$

LEMMA 5.2. Let $X \in U_{x} M$. We have

$$
\begin{gathered}
\eta(X)=H(X, X)+\beta \xi(X), \\
(D \eta)(X)=\alpha(D \xi)(X),
\end{gathered}
$$

where $\alpha=-\left(\lambda_{1}^{2}+\lambda_{2}^{2}+\lambda_{3}^{2}\right) / 2$ and $\beta=\alpha-\lambda_{1} \lambda_{2} / a_{3}^{\prime}$.

Proof. From (5.1) we find

$$
\left(D^{2} \xi\right)(X)=(1-b)\left(D^{2} H\right)\left(X^{4}\right)+a_{4}\left(\lambda_{1} \lambda_{2} \lambda_{3}\right)^{-1}\left(D^{4} H\right)\left(X^{6}\right) .
$$

We proved $\left(D^{3} H\right)\left(X^{5}\right)=-\left(\lambda_{2}^{2}+\lambda_{3}^{2}\right)(D H)\left(X^{3}\right)$ in [8, Theorem 3.4, p. 70] (also see [8, Corollary 3.5, p. 71]). Using (5.5), (5.6) and (5.9), we thus have

$$
\left(D^{2} H\right)\left(X^{4}\right)=\frac{1}{2 a}\left(\lambda_{2}^{2}+\lambda_{3}^{2}-\lambda_{1}^{2}\right)\left(D^{2} \xi\right)(X),
$$

so that (5.7) implies

$$
\left(D^{2} H\right)\left(X^{4}\right)=\frac{1}{2 a}\left(\lambda_{2}^{2}+\lambda_{3}^{2}-\lambda_{1}^{2}\right)(\eta(X)-a H(X, X)) .
$$

We now substitute this equation into (5.1). Here we note that

$$
a^{2}=\left(\lambda_{2}^{2}+\lambda_{3}^{2}-\lambda_{1}^{2}\right) /\left\{\left(\lambda_{2}^{2}+\lambda_{3}^{2}-\lambda_{1}^{2}\right)^{2}+4 \lambda_{1}^{2} \lambda_{2}^{2}\right\}
$$

is derived from (5.3) and (5.9). The straightforward computation shows

$$
\xi(X)=\frac{\left\{\left(\lambda_{1}^{2}+\lambda_{2}^{2}+\lambda_{3}^{2}\right) a-\left(\lambda_{2}^{2}+\lambda_{3}^{2}-\lambda_{1}^{2}\right)\right\}(H(X, X)-\eta(X))}{2 a \lambda_{1}^{2} \lambda_{3}^{2}} .
$$

By definition and (5.9) we have

$$
\beta=-\left\{\left(\lambda_{1}^{2}+\lambda_{2}^{2}+\lambda_{3}^{2}\right) a+\left(\lambda_{2}^{2}+\lambda_{3}^{2}-\lambda_{1}^{2}\right)\right\} / 2 a .
$$

Thus the coefficient of the above equation is equal to $-1 / \beta$. Thus $(5.10)$ has been proved. Since $(D \eta)(X)=(D H)\left(X^{3}\right)+\beta(D \xi)(X)$, (5.11) clearly follows from (5.2). Q.E.D.

Since we have

$$
\begin{array}{r}
\langle\xi(X), \eta(X)\rangle=\left\langle\xi(X), a H(X, X)+\left(D^{2} \xi\right)(X)\right\rangle \\
=a(b-a)-\left(1-a^{2}\right)=a b-1, \\
\langle\xi(X), H(X, X)+\beta \xi(X)\rangle=b-a+\beta\left(1-b^{2}\right)
\end{array}
$$


for $X \in U_{x} M,(5.10)$ gives

$$
\beta=(a-1) /(1-b)<0 .
$$

Lemma 5.3. Let $X \in U_{x} M, Z \in \mathscr{H}_{x}^{*}(X)$ and $Y \in T_{x} \operatorname{Cut}(y)$. Then we obtain

$$
\begin{aligned}
\delta_{1}(D \xi)_{X}(Z)- & \frac{1}{a}(\alpha-\beta) \xi_{X}\left(A_{(D \xi)(X)} Z\right) \\
& =-3\left\{2 H\left(X, A_{(D \xi)(X)} Z\right)+(D \xi)_{X}\left(A_{H(X, X)} Z\right)\right\}, \\
\delta_{2}(D \xi)_{X}(Y)- & \frac{1}{a}(\alpha-\beta) \xi_{X}\left(A_{(D \xi)(X)} Y\right) \\
= & -3\left\{2 H\left(X, A_{(D \xi)(X)} Y\right)+(D \xi)_{X}\left(A_{H(X, X)} Y\right)\right\},
\end{aligned}
$$

where $\delta_{1}=(\alpha-\beta)(a+1)+3(\beta+1)$ and $\delta_{2}=(\alpha-\beta) a+3(\beta+1)$.

Proof. Differentiate (2.3) with respect to $s$ three times. Then at $s=L$ we have

$$
\begin{aligned}
& 3 a(D \eta)(V ; X)+f_{1}^{\prime \prime \prime}(L)(D \xi)(V ; X) \\
& =-\xi_{X}\left(A_{(D \eta)(X)} V\right)-3 \eta_{X}\left(A_{(D \xi)(X)} V\right) \\
& \quad-3 a(D \xi)_{X}\left(V+\frac{1}{a} A_{\eta(X)} V\right)+(D \eta)_{X}\left(b V-A_{\xi(X)} V\right)
\end{aligned}
$$

for $V \in\{X\}^{\perp}$. So by Lemma 5.2,

$$
\begin{gathered}
\{a(\alpha-\beta)+3 a-b \alpha\}(D \xi)_{X}(V)+(3 \beta+\alpha)(D \xi)_{X}\left(A_{\xi(X)} V\right) \\
+\left(3 a \beta+f_{1}^{\prime \prime \prime}(L)\right)(D \xi)(V ; X)+(3 \beta+\alpha) \xi_{X}\left(A_{(D \xi)(X)} V\right) \\
=-3\left\{2 H\left(X, A_{(D \xi)(X)} V\right)+(D \xi)_{X}\left(A_{H(X, X)} V\right)\right\} .
\end{gathered}
$$

It is easily verified that $f_{1}^{\prime \prime \prime}(L)=-\lambda_{1}^{2} a+\lambda_{1} \lambda_{2} a_{3}^{\prime}$. Using (5.9), we have $\alpha=-\lambda_{1}^{2}-$ $\lambda_{1} \lambda_{2} a / a_{3}^{\prime}$, and hence $f_{1}^{\prime \prime \prime}(L)=\alpha a+\lambda_{1} \lambda_{2} a^{2} / a_{3}^{\prime}+\lambda_{1} \lambda_{2} a_{3}^{\prime}=\alpha a+\alpha-\beta$. When $V=Z$, the above equation reduces to (5.13), where we have used Theorem 3.4, (5.12) and the equation

$$
(D \xi)(Z ; X)=(D \xi)_{X}(Z)-\frac{1}{a} \xi_{X}\left(A_{(D \xi)(X)} Z\right)
$$

(cf. Proposition 3.6). When $V=Y$, we have (5.14) in a similar way. Q.E.D.

If $a_{4}=0$, then (5.6) and (5.9) give $a=\left(\lambda_{2}^{2}+\lambda_{3}^{2}-\lambda_{1}^{2}\right) /\left(\lambda_{1}^{2}+\lambda_{2}^{2}+\lambda_{3}^{2}\right)$, which means that $\xi(X)=0$ (see the proof of Lemma 5.2), so we have $b=1$. This contradicts the assumption that $f$ is an embedding. Thus we see that $a_{4} \neq 0$.

Let $h(s), k(s), l(s)$ be defined by

$$
\begin{aligned}
& h(s)=1-F(s)-(1-b) f_{4}(s) / a_{4}, \\
& k(s)=f_{4}(s) / a_{4}, \quad l(s)=f_{3}(s) / a_{3}^{\prime} .
\end{aligned}
$$

Since

$$
\xi(s ; X)=(1-F(s)) H(X, X)+f_{4}(s)\left(\lambda_{1} \lambda_{2} \lambda_{3}\right)^{-1}\left(D^{2} H\right)\left(X^{4}\right)
$$

and

$$
\zeta(s ; X)=f_{3}(s)\left(\lambda_{1} \lambda_{2}\right)^{-1}(D H)\left(X^{3}\right),
$$

we can represent them by

$$
\begin{gathered}
\xi(s ; X)=h(s) H(X, X)+k(s) \xi(X), \\
\zeta(s ; X)=l(s)(D \xi)(X)
\end{gathered}
$$


for every $X \in U_{x} M$, which are shown by using (5.1) and (5.2). Then (2.3) is rewritten as

$$
\begin{aligned}
\left\{\frac{1}{3}(\alpha-\beta) f_{1} h-F l\right\}(D \xi)_{X}(V)+k l(D \xi)_{X}\left(A_{\xi(X)} V\right) \\
+f_{1} k(D \xi)(V ; X)+k l \xi_{X}\left(A_{(D \xi)(X)} V\right) \\
=-h l\left\{2 H\left(X, A_{(D \xi)(X)} V\right)+(D \xi)_{X}\left(A_{H(X, X)} V\right)\right\}
\end{aligned}
$$

for every $V \in\{X\}^{\perp}$. If $V=Z \in \mathscr{H}_{x}^{*}(X)$, then this equation reduces to

$$
\begin{aligned}
\left\{\frac{1}{3}(\alpha-\beta) f_{1} h-F l+f_{1} k+(b-a) k l\right\}(D \xi)_{X}(Z) & \\
& +\left(k l-\frac{1}{a} f_{1} k\right) \xi_{X}\left(A_{(D \xi)(X)} Z\right) \\
= & -h l\left\{2 H\left(X, A_{(D \xi)(X)} Z\right)+(D \xi)_{X}\left(A_{H(X, X)} Z\right)\right\} .
\end{aligned}
$$

If $V=Y \in T_{x} \operatorname{Cut}(y)$, then we obtain

$$
\begin{aligned}
\left\{\frac{1}{3}(\alpha-\beta) f_{1} h-F l+b k l\right\}(D \xi)_{X}(Y) & \\
& +\left(k l-\frac{1}{a} f_{1} k\right) \xi_{X}\left(A_{(D \xi)(X)} Y\right) \\
= & -h l\left\{2 H\left(X, A_{(D \xi)(X)} Y\right)+(D \xi)_{X}\left(A_{H(X, X)} Y\right)\right\} .
\end{aligned}
$$

Lemma 5.4. Let $X \in U_{x} M, Z \in \mathscr{H}_{x}^{*}(X)$ and $Y \in T_{x} \operatorname{Cut}(y)$. Then we have

$$
\begin{gathered}
p(D \xi)_{X}(Z)+q \xi_{X}\left(A_{(D \xi)(X)} Z\right)=0, \\
(p+a q)(D \xi)_{X}(Y)+q \xi_{X}\left(A_{(D \xi)(X)} Y\right)=0,
\end{gathered}
$$

where functions $p$ and $q$ are defined by $p=\frac{1}{2}(\alpha-\beta) f_{1} h-F l+f_{1} k+(b-a) k l$ $-\frac{1}{3} \delta_{1} h l$ and $q=k l-\frac{1}{a} f_{1} k+\frac{1}{3 a}(\alpha-\beta) h l$.

Proof. These equations are easily derived from (5.13), (5.14), (5.17) and (5.18). Q.E.D.

LEMMA 5.5. The Frenet matrix $\Lambda$ can be normalized as

$$
\left(\begin{array}{cccc}
0 & -\nu & & \\
\nu & 0 & & \\
& & 0 & -2 \nu \\
& & 2 \nu & 0
\end{array}\right)
$$

where $\nu=\sqrt{-\beta}$.

Proof. Suppose that $e \geqslant 2$. Taking account of Lemma 3.2 into (5.19), we see that $p$ vanishes identically since $\left\|(D \xi)_{X}(Z)\right\|^{2}=1-a^{2} \neq 0$ for $Z \in \mathscr{H}_{x}^{*}(X) \cap U_{x} M$. Next suppose $e=1$. From (5.20) we have

$$
\begin{aligned}
0= & \left\langle(p+a q)(D \xi)_{X}(Y)+q \xi_{X}\left(A_{(D \xi)(X)} Y\right), H(X, Y)\right\rangle \\
= & -(p+a q)\langle(D \xi)(X), H(Y, Y)\rangle \\
& -b q\left\langle A_{(D \xi)(X)} Y, Y\right\rangle+(b-a) q\left\langle A_{(D \xi)(X)} Y, Y\right\rangle \\
= & -(p+2 a q)\left\langle A_{(D \xi)(X)} Y, Y\right\rangle
\end{aligned}
$$


for $Y \in T_{x} \operatorname{Cut}(y)$. Assume that $p+2 a q$ does not vanish identically. Then $A_{(D \xi)(X)} Y$ $=0$, where we note that $A_{(D \xi)(X)} X=0$. Thus also (5.20) shows $(p+a q)(D \xi)_{X}(Y)$ $=0$. If $(D \xi)_{X}(Y) \neq 0$ for some $Y \in T_{x} \operatorname{Cut}(y)=\{X\}^{\perp}$, then $p+a q$ vanishes identically. If $(D \xi)_{X}(Y)=0$ for every $Y \in\{X\}^{\perp}$, then $(D \xi)(X)$ is a constant vector on $U_{x} M$ and hence $(D \xi)(X)=0$, which contradicts $a^{2} \neq 1$. We have shown that $p+2 a q$ or $p+a q$ vanishes identically when $e=1$. We shall compute the fifth derivatives of $p$ and $q$ at $s=0$. Making use of the differential equation satisfied by $f_{i}$, we have the following data:

$$
\begin{aligned}
& f_{1}^{(1)}(0)=1, \quad f_{1}^{(3)}(0)=-\lambda_{1}^{2}, \\
& F(0)=1, \quad F^{(2)}(0)=-1, \quad F^{(4)}(0)=\lambda_{1}^{2}, \\
& h(0)=0, \quad h^{(2)}(0)=1, \quad h^{(4)}(0)=-\lambda_{1}^{2}-(1-b) \lambda_{1} \lambda_{2} \lambda_{3} / a_{4}, \\
& k(0)=0, \quad k^{(2)}(0)=0, \quad k^{(4)}(0)=\lambda_{1} \lambda_{2} \lambda_{3} / a_{4}, \\
& l^{(1)}(0)=0, \quad l^{(3)}(0)=\lambda_{1} \lambda_{2} / a_{3}^{\prime}, \\
& l^{(5)}(0)=-\lambda_{1} \lambda_{2}\left(\lambda_{1}^{2}+\lambda_{2}^{2}+\lambda_{3}^{2}\right) / a_{3}^{\prime},
\end{aligned}
$$

where we note that $f_{i}^{\prime}(0)=0$ if $i \neq 1$. If $\gamma$ is a geodesic parametrized by arc length, then $\langle\gamma(s), \gamma(t)\rangle=F(s-t)$. It follows that $f_{1}(s-L)=\langle\gamma(s), \dot{\gamma}(L)\rangle=a f_{1}(s)$ $+a_{3}^{\prime} f_{3}(s)$. Integrating both sides from 0 to $L$, we have

$$
b-1=a(1-b)+a_{3}^{\prime} \int_{0}^{L} f_{3} d s .
$$

Since $f_{4}^{\prime}=\lambda_{3} f_{3}$, we see that $\int_{0}^{L} f_{3} d s=\lambda_{3}^{-1} a_{4}$. Therefore

$$
a_{3}^{\prime} a_{4}=-(1-b)(1+a) \lambda_{3} .
$$

Using this relation, $\lambda_{1}^{2}=-\{(\alpha-\beta) a+\alpha\}$ and $\lambda_{1} \lambda_{2} / a_{3}^{\prime}=\alpha-\beta$, we can rewrite the above data in terms of $\alpha, \beta$ and $a$. For example, $h^{(4)}(0)=2 \alpha-\beta$. Then the routine calculation shows the fifth derivatives of $p$ and $q$ at $s=0$ are given by

$$
p^{(5)}(0)=\frac{2}{3}(\alpha-\beta)(2 \alpha-5 \beta), \quad q^{(5)}(0)=\frac{5}{3 a}(\alpha-\beta)(2 \alpha-5 \beta) .
$$

Note that $\alpha \neq \beta$. If $e \geqslant 2$, then $2 \alpha=5 \beta$. If $e=1$, then $p^{(5)}(0)+j a q^{(5)}(0)=0$ ( $j=1$ or 2 ), and so $2 \alpha=5 \beta$. From the definition of $\alpha$, we have $\lambda_{1}^{2}+\lambda_{2}^{2}+\lambda_{3}^{2}=-5 \beta$. Since $\lambda_{1} \lambda_{2} / a_{3}^{\prime}=\frac{3}{2} \beta$, we have $\lambda_{2}^{2}+\lambda_{3}^{2}-\lambda_{1}^{2}=3 a \beta$ because of (5.9), and $\lambda_{1}^{2} \lambda_{2}^{2}$ $=\frac{9}{4} \beta^{2}\left(1-a^{2}\right)$. These equations imply

$$
\lambda_{1}^{2}=-\frac{\beta}{2}(3 a+5), \quad \lambda_{2}^{2}=-\frac{9}{2} \beta \frac{1-a^{2}}{3 a+5}, \quad \lambda_{3}^{2}=-\frac{8 \beta}{3 a+5} .
$$

It is easily verified that the characteristic polynomial of $\Lambda$ is given by

$$
x^{4}+\left(\lambda_{1}^{2}+\lambda_{2}^{2}+\lambda_{3}^{2}\right) x^{2}+\lambda_{1}^{2} \lambda_{3}^{2},
$$

so that the characteristic equation is given by $x^{4}-5 \beta x^{2}+4 \beta^{2}=0$, which proves the assertion. Q.E.D.

LEMMA 5.6. We have $\nu L=\pi$ and

$$
f_{1}(s)=\frac{1}{4 \nu}\{2(1-a) \sin \nu s+(1+a) \sin 2 \nu s\}
$$




$$
F(s)=1+\frac{3 a-5}{8 \nu^{2}}+\frac{1}{8 \nu^{2}}\{4(1-a) \cos \nu s+(1+a) \cos 2 \nu s\} .
$$

Proof. In virtue of Lemma 5.5, $f_{1}$ is a linear combination of $\sin \nu s$ and $\sin 2 \nu s$ (cf. $\S 1)$. Let

$$
f_{1}=(u / \nu) \sin \nu s+(v / 2 \nu) \sin 2 \nu s .
$$

The conditions $f_{1}^{\prime}(0)=1$ and $f_{1}^{(3)}(0)=-\lambda_{1}^{2}$ give $u+v=1$ and $u v^{2}+4 v \nu^{2}=$ $\frac{1}{2} \nu^{2}(3 a+5)$, respectively. It follows that $u=\frac{1}{2}(1-a), v=\frac{1}{2}(1+a)$, which show (5.22). Since $F^{\prime}=-f_{1}$ and $F(0)=1$, we have (5.23). It is easy to show $\nu L=\pi$. Q.E.D.

THEOREM 5.7. Let $f: M \rightarrow S(1)$ be a helical minimal embedding of order 4 of a compact Riemannian manifold $M$ into $S(1)$. Then $a>0$ if and only if there is a unique real number $s_{0} \in(0, L)$ such that $f_{1}\left(s_{0}\right)=0$. Assume that $a>0$. Then $a=$ $(e+2) /(n+2)$ and $M$ is isometric to one of $\mathbf{R} P^{n}, \mathbf{C} P^{m}, \mathbf{Q} P^{m}(m \geqslant 2)$, or $\mathbf{C a y} P^{2}$ with standard metrics. If $M=\mathbf{R} P^{n}$, then the curvature is equal to $n / 4(n+3)$. If $M=\mathbf{C} P^{m}, \mathbf{Q} P^{m}$, or $\mathbf{C} a y P^{2}$, then the maximal curvature is equal to $n /(n+e+2)$. And, moreover, $f$ is equivalent to a standard minimal embedding $(c f .[2,11,12])$.

Proof. From (5.22) we have

$$
f_{1}(s)=(1 / 2 \nu) \sin t\{(1-a)+(1+a) \cos t\},
$$

where $t=\nu s$. It follows that $a>0$ if and only if there is $t_{0}$ (uniquely) such that $t_{0} \in(0, \pi), \cos t_{0}=-(1-a) /(1+a)$. Suppose $a>0$. (3.2) implies that $n F-f_{1}^{\prime}$ vanishes at $t=t_{0}$. Thus by Lemma 5.6 we have

$$
\begin{aligned}
& n\left(3 a-5+8 \nu^{2}\right)+4(1-a)\left(n-\nu^{2}\right) \cos t_{0} \\
& +(1+a)\left(n-4 \nu^{2}\right)\left(2 \cos ^{2} t_{0}-1\right)=0 .
\end{aligned}
$$

Substitute $\cos t_{0}=-(1-a) /(1+a)$ into this equation. Using Corollary 3.5 and (5.12), we easily obtain $a=(e+2) /(n+2)$.

Let $X \in U_{x} M$ and let $\gamma$ be the geodesic such that $\gamma(0)=x$, and $\dot{\gamma}(0)=X$. We note that $\left\{J_{V}(s): V \in\{X\}^{\perp}\right\}$ spans the subspace $\{\dot{\gamma}(s)\}^{\perp}$ on $(0, L)$. Thus, at $s_{0}=t_{0} / \nu$, $\{\dot{\gamma}(s)\}^{\perp}$ is parallel to the normal space at $x$ in the Euclidean space $E$ by virtue of (2.1). So we see from (2.2) that $F\left(s_{0}\right) I-A_{\xi\left(s_{0} ; X\right)}-A_{\zeta\left(s_{0} ; X\right)}=0$ on $\{X\}^{\perp}$ where $I$ denotes the identity transformation of $\{X\}^{\perp}$. Exchange $X$ for $-X$. Then we have $F\left(s_{0}\right) I-A_{\xi\left(s_{0} ; X\right)}+A_{\xi\left(s_{0} ; X\right)}=0$ on $\{X\}^{\perp}$. Thus $F\left(s_{0}\right) I-A_{\xi\left(s_{0} ; X\right)}=0$ and $A_{\zeta\left(s_{0} ; X\right)}$ $=0$ on $\{X\}^{\perp}$. Since $f_{1}^{\prime \prime}=-\lambda_{1}^{2} f_{1}+\lambda_{1} \lambda_{2} f_{3}$, using (5.21) (5.22) we see that $l(s)=$ $-(2 \sin \nu s-\sin 2 \nu s) / 4 \nu<0$ on $(0, L)$. It follows from (5.2) and (5.16) that $A_{(D H)(X, X, X)}=0$, and so $\left\langle H\left(X_{1}, X_{2}\right),(D H)\left(X_{3}, X_{4}, X_{5}\right)\right\rangle=0$ for every $X_{i}(i=$ $1, \ldots, 5)$. Recall the Gauss structure equation of $f$ :

$$
R(U, V) W=\langle V, W\rangle U-\langle U, W\rangle V+A_{H(V, W)} U-A_{H(U, W)} V .
$$

Thus, we conclude that $M$ is locally symmetric. We next make use of $F\left(s_{0}\right) I-$ $A_{\xi\left(s_{0} ; X\right)}=F\left(s_{0}\right) I-h\left(s_{0}\right) A_{H(X, X)}-k\left(s_{0}\right) A_{\xi(X)}=0$ on $\{X\}^{\perp}$. As $f_{1}^{\prime}=1-\lambda_{1} f_{2}$ and $f_{3}^{\prime}=\lambda_{2} f_{2}-\lambda_{3} f_{4}$, we can compute $f_{2}$ and $f_{4}$. From this computation we have

$$
h(s)=(1-\cos 2 \nu s) / 4 \nu^{2}, \quad k(s)=\frac{1}{4}(1-\cos \nu s)^{2} .
$$


Thus we see that

$$
A_{H(X, X)} Z=[\{n(n-e)-4(e+2)\} / 2(n+2)(n+e+2)] Z
$$

for $Z \in \mathscr{H}_{x}^{*}(X)$ and

$$
A_{H(X, X)} Y=[-(e+2)(n+4) / 2(n+2)(n+e+2)] Y
$$

for $Y \in T_{x} \operatorname{Cut}(y)$. Since $f$ is isotropic (cf. [8, Proposition 2.1, p. 66]), we have

$$
R(U, X) X=\left(1-\frac{1}{2} \kappa_{1}^{2}\right) U+\frac{3}{2} A_{H(X, X)} U
$$

for $U \in\{X\}^{\perp}$. Therefore, if $e \geqslant 2$, then the symmetric linear transformation $R(\cdot, X) X$ of $\{X\}^{\perp}$ has two distinct eigenvalues $n /(n+e+2)$ and $n / 4(n+e+2)$ whose eigenspaces are $\mathscr{H}_{x}^{*}(X)$ and $T_{x} \operatorname{Cut}(y)$, respectively. In this case, we see from [2, Theorem 7.23] and Theorem 1.3 that $M$ is simply connected. Since $M$ is complete and locally symmetric, $M$ is a globally symmetric space (cf. [2, Remark.2.2.13]). We have shown that the minimal (resp. maximal) sectional curvature is equal to $n / 4(n+e+2)$ (resp. $n /(n+e+2))$. It follows that the symmetric space $M$ is of rank one. We conclude that $M$ is isometric to one of $\mathbf{C} P^{m}, \mathbf{Q} P^{m}(m \geqslant 2)$, or Cay $P^{2}$. If $e=1$, then the symmetric linear transformation $R(\cdot, X) X$ is equal to $\{n / 4(n+3)\} I$ and hence all sectional curvatures of $M$ are equal to $n / 4(n+3)$. Using [2, Proposition 5.57] and Theorem 1.3, we conclude that $M$ is isometric to $\mathbf{R} P^{n}$. Finally the assertion that $f$ is equivalent with a standard minimal embedding is due to Tsukada [11, Corollary 2.8]. Q.E.D.

REMARK. It is well known in the theory of harmonic manifolds that if $M$ is a compact locally harmonic manifold and locally symmetric, then $M$ is locally isometric to a compact rank-one symmetric space (cf. [2], [8, Theorem 6.5]) shows that if $f$ : $M \rightarrow S(1)$ is a helical minimal immersion, then $M$ is globally harmonic. Thus if we make use of these results, then, in the proof of the above theorem, it suffices to show that $A_{(D H)\left(X^{3}\right)}=0$.

6. Weinstein integer. In Theorem 5.7 we assumed $a>0$ to show that $M$ is isometric to a compact rank-one symmetric space. The reason is that we needed $s_{0} \in(0, L)$ such that $f_{1}\left(s_{0}\right)=0$. In this section we shall study the Weinstein integer (cf. [2, Theorem 2.21, p. 59, and 13]) of the compact Riemannian manifold $M$ which admits a helical minimal embedding of order 4 into a unit sphere $S(1)$ and show that if the Weinstein integer $i(M)$ of $M$ is equal to that of a compact rank-one symmetric space, then $a>0$. Here we recall the definition of the Weinstein integer. If $(N, g)$ is an $n$-dimensional $C_{2 L}$-manifold (a manifold all of whose geodesics are periodic geodesics with the same length $2 L$ ), then the ratio

$$
\frac{\operatorname{Vol}(N, g)}{\operatorname{Vol}\left(S^{n}, \operatorname{can}\right)}\left(\frac{\pi}{L}\right)^{n}
$$

is an integer $i(N, g)$ called the Weinstein integer, where can denotes the standard metric with curvature 1 and

$$
\operatorname{Vol}\left(S^{n}, \operatorname{can}\right)=\frac{2 \pi^{(n+1) / 2}}{\Gamma((n+1) / 2)} .
$$


In the present situation, we have

$$
i(M)=\frac{\operatorname{Vol}(M)}{\operatorname{Vol}\left(S^{n}, \operatorname{can}\right)} \nu^{n} .
$$

Since

$$
\begin{aligned}
\operatorname{Vol}(M) & =\int_{U_{x} M} \int_{0}^{L} s^{n-1} \Theta(s X) d s d \sigma \\
& =\operatorname{Vol}\left(S^{n-1}, \operatorname{can}\right) \int_{0}^{L} s^{n-1} \Theta(s) d s,
\end{aligned}
$$

where $\sigma$ denotes the canonical measure on the unit sphere $U_{x} M$, we have from (6.1)

$$
i(M)=\frac{\Gamma((n+1) / 2)}{\pi^{1 / 2} \Gamma(n / 2)} \nu^{n} \int_{0}^{L} s^{n-1} \Theta(s) d s .
$$

Proposition 6.1. The Weinstein integer $i(M)$ of $M$ is given by

$$
i(M)=2^{n-1} \frac{\Gamma((n+1) / 2) \Gamma(e / 2)}{\pi^{1 / 2} \Gamma((n+e) / 2)} F\left(\frac{n}{2}, \chi, \frac{n+e}{2}, a+1\right),
$$

where $F(\cdot, \cdot, \cdot, \cdot)$ denotes the hypergeometric function (cf. [14]) and

$$
\chi=\{(e+2)-(n+2) a\} / 2(1-a) .
$$

Proof. From (3.2) we have

$$
\left(\log s^{n-1} \Theta(s)\right)^{\prime}=\left(n F(s)-f_{1}^{\prime}(s)\right) / f_{1}(s) \equiv \omega(s) .
$$

Thus we have

$$
s^{n-1} \Theta(s)=C \exp \int_{0}^{s} \omega(u) d u,
$$

$C$ being a certain constant. If we put $t=\nu u$, then by Lemma 5.6

$$
\begin{aligned}
& \omega(u)=\frac{1}{2 \nu}\left[\frac{n\left(3 a-5+8 \nu^{2}\right)}{2(1-a) \sin t+(1+a) \sin 2 t}\right. \\
&\left.+\frac{4(1-a)\left(n-\nu^{2}\right) \cos t+(1+a)\left(n-4 \nu^{2}\right) \cos 2 t}{2(1-a) \sin t+(1+a) \sin 2 t}\right] .
\end{aligned}
$$

A long but routine calculation shows

$$
s^{n-1} \Theta(s)=\left.C|1-a+(1+a) \cos t|^{-\chi}(\sin (t / 2))^{n-1}(\cos (t / 2))^{e-1}\right|_{t=\nu s} .
$$

Since $\Theta(0)=1$, we see that

$$
\begin{aligned}
s^{n-1} \Theta(s)= & (2 / \nu)^{n-1} 2^{x}\{(1+a) \cos \nu s+1-a\}^{-\chi} \\
& \cdot(\sin (\nu s / 2))^{n-1}(\cos (\nu s / 2))^{e-1} .
\end{aligned}
$$

Here we note that if $a>0$, then $a=(e+2) /(n+2)$, and hence $\chi=0$. Substitute (6.3) into (6.2). Then we have

$$
\begin{aligned}
i(M)= & \frac{\Gamma((n+1) / 2)}{\pi^{1 / 2} \Gamma(n / 2)} \\
& \cdot 2^{n-1+\chi} \int_{0}^{\pi}\{(1+a) \cos t+1-a\}^{-\chi}\left(\sin \frac{t}{2}\right)^{n-1}\left(\cos \frac{t}{2}\right)^{e-1} d t .
\end{aligned}
$$


Noting that

$$
(1+a) \cos t+1-a=2\left(\cos ^{2}(t / 2)-a \sin ^{2}(t / 2)\right),
$$

we integrate by substitution $u=\sin ^{2}(t / 2)$. Then we obtain

$$
i(M)=\frac{\Gamma((n+1) / 2)}{\pi^{1 / 2} \Gamma(n / 2)} 2^{n-1} \int_{0}^{1}\{1-(a+1) u\}^{-\chi} u^{n / 2-1}(1-u)^{e / 2-1} d u .
$$

Thus, the formula

$$
F(a, b, c, z)=\frac{\Gamma(c)}{\Gamma(a) \Gamma(c-a)} \int_{0}^{1} u^{a-1}(1-u)^{c-a-1}(1-z u)^{-b} d u,
$$

where $a, b, c$ and $z$ are complex numbers such that $\mathscr{R} c>\mathscr{R} a>0$ and $|z|<1$ (cf. [14, p. 293]) gives the assertion. Q.E.D.

If $a>0$, then $\chi=0$. Since $F(n / 2,0,(n+e) / 2, a+1)=1$, the condition $a>0$ implies that

$$
i(M)=2^{n-1} \Gamma((n+1) / 2) \Gamma(e / 2) / \pi^{1 / 2} \Gamma((n+e) / 2) .
$$

We can see that this number is equal to the Weinstein integer of a compact rank-one symmetric space. For instance, if $e=2$, then

$$
i(M)=2^{n-1} \frac{\Gamma(m+1 / 2)}{\pi^{1 / 2} \Gamma(m+1)}=2^{2 m-1} \frac{(2 m) ! \pi^{1 / 2}}{\pi^{1 / 2}(m !)^{2} 2^{2 m}}=\left(\begin{array}{c}
2 m-1 \\
m-1
\end{array}\right),
$$

which is the Weinstein integer of $\mathbf{C} P^{m}$ with the canonical metric (see [2, p. 60]). Conversely, we have

THEOREM 6.2. Assume that a compact $n$-dimensional Riemannian manifold $M$ admits $a$ helical minimal embedding of order 4 into a unit sphere. If $i(M)=i\left(\mathbf{R} P^{n}\right.$, can) $(e=1)$, then $M$ is isometric to $\mathbf{R} P^{n}$ of constant curvature $n / 4(n+3)$. If $i(M)=$ $i\left(\mathbf{C} P^{m}, \mathrm{can}\right)(e=2), i\left(\mathbf{Q} P^{m}, \mathrm{can}\right)(e=4)$ and $i\left(\mathbf{C a y} P^{2}, \mathrm{can}\right)(e=8)$, then $M$ is isometric to $\mathbf{C} P^{m}, \mathbf{Q} P^{m}$ and $\mathbf{C}$ ay $P^{2}$, respectively, with standard metrics of maximal curvature $n /(n+e+2)$.

Proof. If $a<0$, then $\chi>0$, and hence

$$
F(n / 2, \chi,(n+e) / 2, a+1)>1,
$$

where we note that $a>-1$. By Proposition 6.1 we see that

$$
i(M)>2^{n-1} \Gamma\left(\frac{n+1}{2}\right) \Gamma\left(\frac{e}{2}\right) / \pi^{1 / 2} \Gamma\left(\frac{n+e}{2}\right) .
$$

This contradicts the assumption. Thus we have $a>0$. The assertion follows from Theorem 5.7. Q.E.D.

Unfortunately, at present we can not prove $a>0$ without condition $i(M)=i$ (compact rank-one symmetric space).

REMARK. Since $M$ is a globally harmonic manifold, if $e=1$, then we see from [2, Proposition 5.57, p. 142 and Corollary D.3, p. 236] that $M$ is isometric to $\mathbf{R} P^{n}$ of constant curvature. Thus in Theorem 6.2 the condition $i(M)=i\left(\mathbf{R} P^{n}\right)$ is redundant. 


\section{REFERENCES}

1. A. Allamigeon, Propriétés globales des espaces de Riemann harmoniques, Ann. Inst. Fourier (Grenoble) 15 (1965), 91-132.

2. A. Besse, Manifolds all of whose geodesics are closed, Ergeb. Math Grenzgeb., Band 93, SpringerVerlag, Berlin and New York, 1978.

3. R. Escobales, Riemannian submersions with totally geodesic fibers, J. Differential Geom. 10 (1975), 253-276.

4. W. Klingenberg, Riemannian geometry, Gruyter Stud. in Math., vol. 1; de Gruyter, Berlin, 1982.

5. J. A. Little, Manifolds with planar geodesics, J. Differential Geom. 11 (1976), 265-285.

6. H. Nakagawa, On a certain minimal immersion of a Riemannian manifold into a sphere, Kōdai Math. J. 3 (1980), 321-340.

7. K. Sakamoto, Planar geodesic immersions, Tôhoku Math. J. 29 (1977), 25-56.

8. __ Helical immersions into a unit sphere, Math. Ann. 26 (1982), 63-80.

9. On a minimal helical immersions into a unit sphere, Geometry of Geodesics and Related Topics, Advanced Studies in Pure Math. 3 (1984), 193-211.

10. K. Tsukada, Isotropic minimal immersions of spheres into spheres, J. Math. Soc. Japan 35 (1983), 355-379.

11. Helical geodesic immersions of compact rank one symmetric spaces into spheres, Tokyo J. Math. 6 (1983), 267-285.

12. N. R. Wallach, Symmetric spaces, (W. M. Boothby and G. L. Weiss, Eds.), Marcel Dekker, New York, 1972.

13. A. Weinstein, On the volume of manifolds all of whose geodesics are closed, J. Differential Geom. 9 (1974), 513-517.

14. E. T. Whittaker and G. N. Watson, A course of modern analysis, Cambridge Univ. Press, London and New York, 1965.

Department of Mathematics, Tokyo Institute of Technology, Tokyo 152, Japan 